\title{
A higher Albanese map for complex threefolds based on a construction by M. Green
}

by

\author{
Lorenz Schneider (Erlangen)
}

\begin{abstract}
We construct a higher Abel-Jacobi map for 0-cycles on complex threefolds and prove that it can be used to describe Mumford's pull-back of a differential form, and that its image is infinite-dimensional in many cases. However, making a certain assumption, we show that it is not always injective.
\end{abstract}

1. Introduction. According to the Bloch-Beilinson conjectures for algebraic cycles on a smooth projective variety $X$, there should exist a filtration $F^{\bullet} \mathrm{CH}^{p}(X)$ of the Chow group $\mathrm{CH}^{p}(X)$ which satisfies certain properties (see [Bei]). In particular, in the case of 0 -cycles on complex varieties these properties are:

(i) $F^{n+1} \mathrm{CH}_{0}(X)=0$, where $n=\operatorname{dim} X$.

(ii) The filtration $F^{i}$ is stable under correspondences, that is, for $\Gamma \in$ $\mathrm{CH}^{m}(X \times Y), m=\operatorname{dim} Y$, the induced map

$$
\Gamma_{*}: \mathrm{CH}_{0}(X)_{\mathbb{Q}} \rightarrow \mathrm{CH}_{0}(Y)_{\mathbb{Q}}
$$

satisfies

$$
\Gamma_{*}\left(F^{i} \mathrm{CH}_{0}(X)_{\mathbb{Q}}\right) \subset F^{i} \mathrm{CH}_{0}(Y)_{\mathbb{Q}},
$$

where we write $\mathrm{CH}_{0}(X)_{\mathbb{Q}}$ for $\mathrm{CH}_{0}(X) \otimes_{\mathbb{Z}} \mathbb{Q}$.

(iii) The graded pieces of the filtration are governed by the global holomorphic forms and vice versa, i.e. the map

$$
\operatorname{Gr}_{F}^{i} \Gamma_{*}: \operatorname{Gr}_{F}^{i} \mathrm{CH}_{0}(X)_{\mathbb{Q}} \rightarrow \operatorname{Gr}_{F}^{i} \mathrm{CH}_{0}(Y)_{\mathbb{Q}}
$$

is zero if and only if the map

$$
\operatorname{cl}(\Gamma)^{*}: H^{0}\left(Y, \Omega_{Y}^{i}\right) \rightarrow H^{0}\left(X, \Omega_{X}^{i}\right)
$$

is zero.

Key words and phrases: Abel-Jacobi map, threefold, Mumford's pull-back, mixed Hodge structure, higher Jacobian. 
It follows from (iii) that $F^{1} \mathrm{CH}_{0}(X)=\mathrm{CH}_{0}(X)_{\text {hom }}$ and also that

$$
F^{2} \mathrm{CH}_{0}(X)=\mathrm{CH}_{0}(X)_{\mathrm{alb}},
$$

the subgroup of cycles lying in the kernel of the Albanese map.

An attempt to prove these conjectures for 0-cycles on smooth surfaces defined over the complex numbers $\mathbb{C}$ was made by M. Green. In [Gre1] and [Gre2] he defined a map $\psi_{2}^{2}$ from $F^{2} \mathrm{CH}_{0}(S):=\operatorname{ker}\left(\mathrm{alb}_{S}\right)$ to a higher Jacobian $J_{2}^{2}(S)$ and conjectured that it was an isomorphism.

Had this been true, the construction would have in particular given a positive answer to Bloch's conjecture for surfaces, since the Jacobian $J_{2}^{2}(S)$ is built from the transcendental part of $H^{2}(S, \mathbb{Z})$. A filtration on $\mathrm{CH}_{0}(S)$ would have been obtained by setting

$$
\begin{aligned}
& F^{1} \mathrm{CH}_{0}(S)=\mathrm{CH}_{0}(S)_{\text {hom }}, \\
& F^{2} \mathrm{CH}_{0}(S)=\operatorname{ker}\left(\operatorname{alb}_{S}\right), \\
& F^{3} \mathrm{CH}_{0}(S)=\operatorname{ker}\left(\psi_{2}^{2}\right)=0 .
\end{aligned}
$$

Green's construction involves principally extensions of mixed Hodge structures coming from short exact sequences in relative cohomology of a variety and a codimension one subvariety.

However, soon after his paper had appeared C. Voisin published a counterexample in [Voi3] to his claim that $\psi_{2}^{2}$ was an isomorphism. Nevertheless, in the same paper, she shows that Green's higher Abel-Jacobi invariant has the merit of governing Mumford's pull-back of holomorphic 2-forms on the surface $S$ to a variety parametrizing 0-cycles on $S$. Since this pull-back technique remains one of the most important in the study of algebraic cycles, it seems useful to prove an analogous result for threefolds (see Theorem 4.5).

In this article, we construct, for a smooth projective threefold $X$, two maps $\psi_{2}^{3}: \operatorname{ker}\left(\operatorname{alb}_{X}\right) \rightarrow J_{2}^{3}$ and $\psi_{3}^{3}: \operatorname{ker}\left(\psi_{2}^{3}\right) \rightarrow J_{3}^{3}$ from subgroups of $\mathrm{CH}_{0}(X)$ to higher Jacobians (see Definitions 2.2 and 3.10). The first map is essentially Green's map for surfaces. In our construction of the map $\psi_{3}^{3}$ we use pairings derived from the perfect pairings

$$
H^{n_{i}}\left(Y_{i}, \mathbb{Z}\right) \otimes_{\mathbb{Z}} H^{n_{i}}\left(Y_{i}, \mathbb{Z}\right) \rightarrow \mathbb{Z}, \quad n_{i}=\operatorname{dim} Y_{i},
$$

to couple the various one-extension classes and make the construction independent of the chosen subvarieties $Y_{i}$.

If it were to satisfy the conjectures, the map $\psi_{3}^{3}$ should be injective, since the conjectural filtration $F^{\bullet}$ on $\mathrm{CH}_{0}(X)$ should have exactly three steps. However, it can be shown that if $X$ is the product of a curve $C$ with a surface $S$, then for certain 0-cycles on $X, \psi_{3}^{3}$ can be expressed as a product of the Abel-Jacobi map $\mu_{C}$ for divisors on $C$ and Green's map $\psi_{2}^{2}$ for 0-cycles on $S$. So, under a certain assumption, which is given in the final section, Voisin's counterexample also furnishes one in this case. 
Voisin proves that Green's map does indeed have an infinite-dimensional image if $p_{g}(S)>0$, just as Mumford's theorem demands. Analogously, we prove that the map $\psi_{3}^{3}$ for a threefold $X$ also has infinite-dimensional image on $J_{3}^{3}(X)$ if $H^{0}\left(K_{X}\right) \neq 0$ (see Theorem 5.1).

One comment is in order: There are two ways of describing the oneextension classes of mixed Hodge structures. One is due to J. Carlson and involves integral and Hodge splittings of a short exact sequence associated to the long exact sequence of relative cohomology of the pair $\left(Y_{k}, Y_{k-1}\right)$, and the other one uses the image of the (modified) graph $\Gamma_{j}$ of the map $j: Y_{k-1} \rightarrow Y_{k}$ on Griffiths' intermediate Jacobian $J^{2 k-1}\left(Y_{k-1} \times Y_{k}\right)$. We need both versions and use whichever one is more convenient for our purposes in a given context.

An excellent source for many of the objects and techniques used in this paper are the books [Voi1] and [Voi2].

Acknowledgements. This article is taken from my Ph.D. thesis, which was completed under the guidance of Claire Voisin. I would like to thank her for her generosity and support. I also want to thank Jishnu Biswas, Catriona Maclean and Gianluca Pacienza for many useful and exciting discussions, and Chris Peters for his help with the proof of Proposition 5.2. Finally, I would like to thank an anonymous referee for valuable comments and suggestions.

2. Construction of $\psi_{2}^{3}$. We begin by constructing a higher Abel-Jacobi map on $F^{2} \mathrm{CH}_{0}(X):=\operatorname{ker}\left(\operatorname{alb}_{X}\right)$ for a threefold $X$, which is immediately derived from Green's map for surfaces. First of all we explain how his map is defined.

2.1. Green's higher Abel-Jacobi map. Let $S$ be a smooth projective complex surface, and let $Z_{0}$ be a 0 -cycle of $S$ in the kernel of the Albanese map. Let $i: C \rightarrow S$ be a smooth, but not necessarily connected curve, which maps generically one-to-one onto its image $i(C)$ on $S$, and let $Z$ be a 0 -cycle supported on $C$, of degree 0 on each component of $C$, such that $i_{*}(Z)=Z_{0}$.

Green constructs two Abel-Jacobi invariants $f_{C, Z}$ and $e_{S, C}$. The first is just the image of $Z$ under the Abel-Jacobi map $\mu_{C}: \mathrm{CH}_{0}(C)_{0} \rightarrow J(C)$, where $J(C)=H^{0,1}(C) / H^{1}(C, \mathbb{Z})$ is the Jacobian of $C$ and $\mathrm{CH}_{0}(C)_{0}=$ $\mathrm{CH}_{0}(C)_{\text {hom }}$. However, here this Jacobian is interpreted as a real torus

$$
J(C) \cong H^{1}(C, \mathbb{Z}) \otimes_{\mathbb{Z}} \mathbb{R} / \mathbb{Z} .
$$

This is done via the isomorphisms $H^{1}(C, \mathbb{R}) \cong H^{0,1}(C)$, obtained as the composite $H^{1}(C, \mathbb{R}) \hookrightarrow H^{1}(C, \mathbb{C}) \rightarrow H^{0,1}(C)$, and $H^{1}(C, \mathbb{R}) / H^{1}(C, \mathbb{Z}) \cong$ $H^{1}(C, \mathbb{Z}) \otimes_{\mathbb{Z}} \mathbb{R} / \mathbb{Z}$. We consider $f_{C, Z}$ as an element of this tensor product.

The second invariant $e_{S, C}$ is defined as the extension class of a short exact sequence of mixed Hodge structures as follows. Let $\tau: S^{\prime} \rightarrow S$ be 
a blow-up of $S$ along the singularities of the image of $C$, i.e. a birational morphism such that $i^{\prime}: C \hookrightarrow S^{\prime}$ is the inclusion map and $\tau \circ i^{\prime}=i$. The long exact sequence in relative cohomology for the couple $i^{\prime}: C \hookrightarrow S^{\prime}$ then leads to the short exact sequence

$$
0 \rightarrow \frac{H^{1}(C, \mathbb{Z})}{i^{\prime *} H^{1}\left(S^{\prime}, \mathbb{Z}\right)} \rightarrow H^{2}\left(S^{\prime}, C, \mathbb{Z}\right) \rightarrow H^{2}\left(S^{\prime}, \mathbb{Z}\right)_{C} \rightarrow 0
$$

where

$$
H^{2}\left(S^{\prime}, \mathbb{Z}\right)_{C}:=\operatorname{ker}\left(i^{*}: H^{2}\left(S^{\prime}, \mathbb{Z}\right) \rightarrow H^{2}(C, \mathbb{Z})\right) .
$$

There is naturally the same sequence with complex coefficients; the important point is that it is a short exact sequence of mixed Hodge structures. Let

$$
H^{2}(S, \mathbb{Z})_{\operatorname{tr}} \subset H^{2}(S, \mathbb{Z})
$$

be the orthogonal of the Néron-Severi group $\operatorname{NS}(S) \subset H^{2}(S, \mathbb{Z})$ (the subgroup generated by classes of curves), i.e. the kernel of the map

$$
\langle\mathrm{NS}(S), \cdot\rangle: H^{2}(S, \mathbb{Z}) \rightarrow \mathbb{Z}
$$

given by the intersection pairing $\langle\cdot, \cdot\rangle$ on $H^{2}(S, \mathbb{Z})$. This is the transcendental part of $H^{2}(S, \mathbb{Z})$ and by definition it remains invariant under birational maps. Since a class in $H^{2}(S, \mathbb{Z})_{\text {tr }}$ naturally vanishes when pulled back via $i^{*}$ to $H^{2}(C, \mathbb{Z})$ and there is an inclusion $\tau^{*} H^{2}(S, \mathbb{Z}) \subset H^{2}\left(S^{\prime}, \mathbb{Z}\right)$, we obtain the inclusion map

$$
t: H^{2}(S, \mathbb{Z})_{\operatorname{tr}} \hookrightarrow H^{2}\left(S^{\prime}, \mathbb{Z}\right)_{C} .
$$

Again, this is also defined for the cohomology groups with complex coefficients, and the inclusion $t$ is actually a morphism of Hodge structures. Also note that $H^{1}\left(S^{\prime}, \mathbb{Z}\right) \cong H^{1}(S, \mathbb{Z})$; but to make the notation easier let us assume for the moment that $S$ is regular (otherwise we just keep writing the quotient on the left).

Now the sequence (1) pulls back via this inclusion map to the sequence

$$
0 \rightarrow H^{1}(C, \mathbb{Z}) \rightarrow H^{2}\left(S^{\prime}, C, \mathbb{Z}\right)_{\operatorname{tr}} \rightarrow H^{2}(S, \mathbb{Z})_{\operatorname{tr}} \rightarrow 0,
$$

where $H^{2}\left(S^{\prime}, C, \mathbb{Z}\right)_{\operatorname{tr}}$ is the fibered product of $H^{2}\left(S^{\prime}, C, \mathbb{Z}\right)$ and $H^{2}(S, \mathbb{Z})_{\operatorname{tr}}$ over $H^{2}\left(S^{\prime}, \mathbb{Z}\right)_{C}$. Because $(2)$ is an exact sequence of mixed Hodge structures, there is a section $\sigma_{\mathbb{Z}}$ of the sequence (2) that preserves the integral structure,

$$
\sigma_{\mathbb{Z}}\left(H^{2}(S, \mathbb{Z})_{\mathrm{tr}}\right) \subset H^{2}\left(S^{\prime}, C, \mathbb{Z}\right)_{\mathrm{tr}},
$$

and a section $\sigma_{F}$ that preserves the Hodge filtration,

$$
\sigma_{F}\left(F^{p} H^{2}(S)_{\mathrm{tr}}\right) \subset F^{p} H^{2}\left(S^{\prime}, C\right)_{\mathrm{tr}} .
$$

The difference of these two sections will be viewed as a map

$$
\psi=\sigma_{F}-\sigma_{\mathbb{Z}}: H^{2}(S, \mathbb{C})_{\operatorname{tr}} \rightarrow H^{1}(C, \mathbb{C}) .
$$


Evidently $\psi$ depends on the choice of the sections, but in the quotient

$$
\begin{aligned}
& J\left(\operatorname{Hom}\left(H^{2}(S)_{\mathrm{tr}}, H^{1}(C)\right)\right. \\
& \quad:=\frac{\operatorname{Hom}_{\mathbb{C}}\left(H^{2}(S, \mathbb{C})_{\mathrm{tr}}, H^{1}(C, \mathbb{C})\right)}{F^{0} \operatorname{Hom}_{\mathbb{C}}\left(H^{2}(S, \mathbb{C})_{\mathrm{tr}}, H^{1}(C, \mathbb{C})\right)+\operatorname{Hom}_{\mathbb{Z}}\left(H^{2}(S, \mathbb{Z})_{\mathrm{tr}}, H^{1}(C, \mathbb{Z})\right)}
\end{aligned}
$$

it does not. This complex torus is isomorphic to $\operatorname{Ext}_{M H S}^{1}\left(H^{2}(S)_{\mathrm{tr}}, H^{1}(C)\right)$ and therefore parametrizes the extensions of the sequence (2). Again we will write it as a real torus: firstly we have

$$
\operatorname{Hom}_{\mathbb{C}}\left(H^{2}(S, \mathbb{C})_{\mathrm{tr}}, H^{1}(C, \mathbb{C})\right) \cong H^{2}(S, \mathbb{C})_{\mathrm{tr}}^{*} \otimes_{\mathbb{C}} H^{1}(C, \mathbb{C})
$$

(and the same for $\mathrm{Hom}_{\mathbb{Z}}$ ) and

$$
F^{0} \operatorname{Hom}_{\mathbb{C}}\left(H^{2}(S, \mathbb{C})_{\mathrm{tr}}, H^{1}(C, \mathbb{C})\right) \cong F^{2}\left(H^{2}(S, \mathbb{C})_{\mathrm{tr}}^{*} \otimes_{\mathbb{C}} H^{1}(C, \mathbb{C})\right),
$$

where in the last expression $F^{\bullet}$ is now the usual Hodge filtration and the right hand side is seen as a Hodge structure of weight 3 . Secondly, just as for the Jacobian of the curve $C$, we may identify

$$
\frac{H^{2}(S, \mathbb{C})_{\mathrm{tr}}^{*} \otimes_{\mathbb{C}} H^{1}(C, \mathbb{C})}{F^{2}\left(H^{2}(S, \mathbb{C})_{\mathrm{tr}}^{*} \otimes_{\mathbb{C}} H^{1}(C, \mathbb{C})\right)} \cong H^{2}(S, \mathbb{R})_{\mathrm{tr}}^{*} \otimes_{\mathbb{R}} H^{1}(C, \mathbb{R})
$$

and then write

$$
\frac{H^{2}(S, \mathbb{R})_{\mathrm{tr}}^{*} \otimes_{\mathbb{R}} H^{1}(C, \mathbb{R})}{H^{2}(S, \mathbb{Z})_{\mathrm{tr}}^{*} \otimes_{\mathbb{Z}} H^{1}(C, \mathbb{Z})} \cong H^{2}(S, \mathbb{Z})_{\mathrm{tr}}^{*} \otimes_{\mathbb{Z}} H^{1}(C, \mathbb{Z}) \otimes_{\mathbb{Z}} \mathbb{R} / \mathbb{Z}
$$

Green considers the extension class $e_{S, C}$ as an element of this tensor product. If we keep track of the irregularity, we get an element $e_{S, C}$ of

$$
H^{2}(S, \mathbb{Z})_{\mathrm{tr}}^{*} \otimes_{\mathbb{Z}} \frac{H^{1}(C, \mathbb{Z})}{H^{1}(S, \mathbb{Z})} \otimes_{\mathbb{Z}} \mathbb{R} / \mathbb{Z}
$$

Now the construction is almost done. Remark that if $Z_{0} \in \operatorname{ker}\left(\operatorname{alb}_{S}\right)$, then the Abel-Jacobi invariant $\mu_{C}(Z)$ must vanish under the map

$$
i_{*}: J(C) \cong H^{1}(C, \mathbb{Z}) \otimes_{\mathbb{Z}} \mathbb{R} / \mathbb{Z} \rightarrow \operatorname{Alb}(S) \cong H^{1}(S, \mathbb{Z}) \otimes_{\mathbb{Z}} \mathbb{R} / \mathbb{Z},
$$

that is, $\mu_{C}(Z) \in \operatorname{ker}\left(i_{*}: H^{1}(C, \mathbb{Z}) \rightarrow H^{1}(S, \mathbb{Z})\right) \otimes_{\mathbb{Z}} \mathbb{R} / \mathbb{Z}$.

Use now the pairing

$$
\operatorname{ker}\left(i_{*}: H^{1}(C, \mathbb{Z}) \rightarrow H^{1}(S, \mathbb{Z})\right) \otimes_{\mathbb{Z}} \frac{H^{1}(C, \mathbb{Z})}{i^{*} H^{1}(S, \mathbb{Z})} \rightarrow \mathbb{Z}
$$

to contract $f_{C, Z}$ and $e_{S, C}$ to an element

$$
f_{C, Z} \cdot e_{S, C} \in H^{2}(S, \mathbb{Z})_{\mathrm{tr}}^{*} \otimes_{\mathbb{Z}} \mathbb{R} / \mathbb{Z} \otimes_{\mathbb{Z}} \mathbb{R} / \mathbb{Z}
$$

Green defines $U_{2}^{2}(S) \subseteq H^{2}(S, \mathbb{Z})_{\mathrm{tr}}^{*} \otimes_{\mathbb{Z}} \mathbb{R} / \mathbb{Z} \otimes_{\mathbb{Z}} \mathbb{R} / \mathbb{Z}$ as the subgroup generated by all elements $f_{C, Z} \cdot e_{S, C}$ such that $i_{*} Z=0$ as a 0 -cycle of $S$. The higher Jacobian $J_{2}^{2}(S)$ is defined as

$$
J_{2}^{2}(S):=\frac{H^{2}(S, \mathbb{Z})_{\mathrm{tr}}^{*} \otimes_{\mathbb{Z}} \mathbb{R} / \mathbb{Z} \otimes_{\mathbb{Z}} \mathbb{R} / \mathbb{Z}}{U_{2}^{2}(S)}
$$


and the map

$$
\psi_{2}^{2}: \mathrm{CH}_{0}(S)_{\mathrm{alb}} \rightarrow J_{2}^{2}(S)
$$

is then defined as follows: if $Z_{0} \in \mathcal{Z}_{0}(S)_{\mathrm{alb}}$, choose a curve $C$, a morphism $j: C \rightarrow S$, and a cycle $Z$ on $C$ homologous to 0 such that $j_{*} Z=Z_{0}$. Then define $\psi_{2}^{2}\left(Z_{0}\right)$ as the image of $f_{C, Z} \cdot e_{S, C}$ in $J_{2}^{2}(S)$. By definition of $U_{2}^{2}$ this image does not depend on the choices of $C$ and $Z$. If now $Z_{0}$ is rationally equivalent to 0 , for some $C$ and $Z$, we may assume that $Z$ is rationally equivalent to 0 in $C$, and it follows that $f_{C, Z}=0$. Hence for this choice $f_{C, Z} \cdot e_{S, C}=0$, which shows that $\psi_{2}^{2}$ defined above on $\mathcal{Z}_{0}(S)$ alb factors through rational equivalence.

2.2. Construction of $\psi_{2}^{3}$. Let $X$ be a smooth projective complex threefold. Let $Z_{0}$ be a 0 -cycle of degree 0 in the kernel of the Albanese map: $\operatorname{alb}_{X}\left(Z_{0}\right)=0 \in \operatorname{Alb}(X)$. Choose a smooth ample surface $S \subset X$ containing $\operatorname{supp}\left(Z_{0}\right)$ : by the Lefschetz hyperplane theorem $H^{1}(X, \mathbb{Z}) \cong H^{1}(S, \mathbb{Z})$, so $\operatorname{Alb}(X) \cong \operatorname{Alb}(S)$ and since $\operatorname{alb}_{X}\left(Z_{0}\right)=0$, we have $\operatorname{alb}_{S}\left(Z_{0}\right)=0$.

Let $i: C \rightarrow S$ be a smooth, but not necessarily connected curve, which maps generically one-to-one onto its image $i(C)$ on $S$, and $Z$ a 0 -cycle supported on $C$ such that $i_{*}(Z)=Z_{0}$.

In general, we will consider all smooth surfaces $j: S \rightarrow X$ and smooth curves $i: C \rightarrow S$ carrying a 0 -cycle $Z$ such that $(j \circ i)_{*}(Z)=Z_{0}$ and $\operatorname{alb}_{S}\left(i_{*}(Z)\right)=0$.

Given such $S, C$ and $Z$ we can apply Green's construction of the higher Abel-Jacobi map $\psi_{2}^{2}$ for a 0 -cycle on a surface. Let now $j_{*}: H^{2}(S, \mathbb{Z}) \rightarrow$ $H^{4}(X, \mathbb{Z})$ be the Gysin morphism induced by $j: S \rightarrow X$ and also let

$$
j_{*}: H^{2}(S, \mathbb{Z})_{\mathrm{tr}}^{*} \rightarrow H^{4}(X, \mathbb{Z}) / j_{*} \mathrm{NS}(S)
$$

be the induced map on the quotient.

Definition 2.1. $H^{4}(X, \mathbb{Z})_{\mathrm{tr}}^{*}=H^{4}(X, \mathbb{Z}) /\left\langle j_{*} \mathrm{NS}(S)\right\rangle$ for all $j: S \rightarrow X$, where $S$ is a smooth surface.

We have the projection

$$
\pi: \frac{H^{4}(X, \mathbb{Z})}{j_{*} \operatorname{NS}(S)} \otimes_{\mathbb{Z}} \mathbb{R} / \mathbb{Z} \otimes_{\mathbb{Z}} \mathbb{R} / \mathbb{Z} \rightarrow H^{4}(X, \mathbb{Z})_{\mathrm{tr}}^{*} \otimes_{\mathbb{Z}} \mathbb{R} / \mathbb{Z} \otimes_{\mathbb{Z}} \mathbb{R} / \mathbb{Z} .
$$

Definition 2.2. Let $U_{2}^{3}(X)$ be the group generated by all $j_{*}\left(e_{S, C} \cdot f_{C, Z}\right)$ for which $(j \circ i)_{*}(Z)=0$ as a 0 -cycle of $X$. Let

$$
J_{2}^{3}(X)=\frac{H^{4}(X, \mathbb{Z})_{\mathrm{tr}}^{*} \otimes_{\mathbb{Z}} \mathbb{R} / \mathbb{Z} \otimes_{\mathbb{Z}} \mathbb{R} / \mathbb{Z}}{U_{2}^{3}(X)} .
$$

LEMma 2.3. The projection of $j_{*}\left(e_{S, C} \cdot f_{C, Z}\right)$ into $J_{2}^{3}(X)$ is independent of the surface $S$ chosen in the construction. 
Proof. Suppose $Z_{1},\left|Z_{1}\right| \subset C_{1} \stackrel{i_{1}}{\rightarrow} S_{1} \stackrel{j_{1}}{\rightarrow} X$, and $Z_{2},\left|Z_{2}\right| \subset C_{2} \stackrel{i_{2}}{\rightarrow} S_{2} \stackrel{j_{2}}{\rightarrow} X$, are both 0 -cycles mapping to $Z_{0}$ on $X$. Then $Z_{1}-Z_{2}$ with

$$
\left|Z_{1}-Z_{2}\right| \subset C_{1} \sqcup C_{2} \stackrel{i^{\prime}}{\rightarrow} S_{1} \sqcup S_{2} \stackrel{j^{\prime}}{\rightarrow} X
$$

obviously satisfies $\left(j^{\prime} \circ i^{\prime}\right)_{*}\left(Z_{1}-Z_{2}\right)=0$, and we have

$$
\begin{aligned}
j_{1 *}\left(e_{S_{1}, C_{1}} \cdot f_{C_{1}, Z_{1}}\right)-j_{2 *} & \left(e_{S_{2}, C_{2}} \cdot f_{C_{2}, Z_{2}}\right) \\
& =j_{*}^{\prime}\left(e_{S_{1} \sqcup S_{2}, C_{1} \sqcup C_{2}} \cdot f_{C_{1} \sqcup C_{2}, Z_{1}-Z_{2}}\right) \in U_{2}^{3}(X) .
\end{aligned}
$$

So, by the independence from the choices made, this defines a map

$$
\psi_{2}^{3}: \operatorname{ker}\left(\operatorname{alb}_{X}\right) \rightarrow J_{2}^{3}(X), \quad Z_{0} \mapsto\left[j_{*}\left(e_{S, C} \cdot f_{C, Z}\right)\right],
$$

which factors through rational equivalence. For if a cycle $Z_{0}$ is rationally equivalent to 0 , then there is a smooth curve $C \stackrel{i}{\rightarrow} X$ and a cycle $Z$ rationally equivalent to 0 in $C$ such that $i_{*} Z=Z_{0}$. Then since $f_{C, Z}=0, i^{\prime}(Z)$ is Albanese equivalent to 0 in $S$, and furthermore we have $j_{*}\left(e_{S, C} \cdot f_{C, Z}\right)=0$ for any $C \stackrel{i^{\prime}}{\rightarrow} S \stackrel{j}{\rightarrow} X$, with $S$ a smooth surface.

3. Construction of $\psi_{3}^{3}$. In this section we construct the higher AbelJacobi map $\psi_{3}^{3}$ defined on $\operatorname{ker}\left(\psi_{2}^{3}\right)$. We consider three extension classes $d_{X, S}, e_{S, C}$ and $f_{C, Z}$, each one reflecting a codimension one subvariety in its ambient variety. The classes $e_{S, C}$ and $f_{C, Z}$ are basically those of Green's construction from the previous section, but in order to contract them with $d_{X, S}$ we exploit the fact that they lead to an invariant that vanishes on $J_{2}^{3}(X)$.

The extension class $d_{X, S}$ introduced here (see Definition 3.7) is not defined via Carlson's method, but instead by considering the graph $\Gamma_{j}$ of the morphism $j: S \rightarrow X$. Modifying it by the $(2,2)$-Künneth component of the cycle class of the diagonal $\Delta_{S} \subset S \times S$ and the (3,3)-component of $\Delta_{X} \subset X \times X$ to obtain a homologically trivial cycle permits us to take its A-J invariant on Griffiths' intermediate Jacobian - however, for a technical reason (basically because the Hodge conjecture is still a conjecture), it does not work quite like this and we have to make a detour through Deligne cohomology in order to carry out our construction.

Then we define the contraction of $d_{X, S}, e_{S, C}$ and $f_{C, Z}$ to $d_{X, S} \cdot e_{S, C} \cdot f_{C, Z}$ and the higher Jacobian $J_{3}^{3}(X)$. This Jacobian is a quotient of $J^{3}(X) \otimes_{\mathbb{Z}}$ $\mathbb{R} / \mathbb{Z} \otimes_{\mathbb{Z}} \mathbb{R} / \mathbb{Z}$, where $J^{3}(X)$ is Griffiths' intermediate Jacobian of $X$, and first of all we must quotient by the image of the Abel-Jacobi map

$$
\phi_{X}: \mathcal{Z}_{1}(X)_{\text {hom }} \rightarrow J^{3}(X)
$$

in order to make this contraction well defined (see Lemma 3.9).

Finally, we show that the map $\psi_{3}^{3}$ is independent of the chosen curve and surface, and that 0 -cycles rationally equivalent to zero are mapped to $(0) \in J_{3}^{3}(X)$. 
Let $Z_{0}$ be a 0 -cycle in the kernel of the map $\psi_{2}^{3}: \operatorname{ker}\left(\operatorname{alb}_{X}\right) \rightarrow J_{2}^{3}(X)$. For an appropriate choice of $Z \rightarrow C \rightarrow S$ we construct invariants

$$
\begin{gathered}
d_{X, S} \in J^{3}(X)_{A J} \otimes_{\mathbb{Z}} H^{2}(S, \mathbb{Z})_{\text {new }}^{*}, \\
e_{S, C} \cdot f_{C, Z} \in H^{2}(S, \mathbb{Z})_{\text {tr,new }}^{*} \otimes_{\mathbb{Z}} \mathbb{R} / \mathbb{Z} \otimes_{\mathbb{Z}} \mathbb{R} / \mathbb{Z},
\end{gathered}
$$

which we contract to an element lying in $J^{3}(X)_{\text {AJ }} \otimes_{\mathbb{Z}} \mathbb{R} / \mathbb{Z}^{\otimes 2}$. This element is then projected onto a certain quotient $J_{3}^{3}(X)$ to obtain our third AbelJacobi invariant of the 0-cycle $Z$.

The invariant $e_{S, C} \cdot f_{C, Z}$ is essentially the one from Green's construction for 0-cycles on surfaces. We show how to obtain $d_{X, S}$. Let $\Gamma_{j} \subset S \times X$ be the graph of the morphism $j: S \rightarrow X$. It has its cohomology class $\left[\Gamma_{j}\right]$ in $H^{6}(S \times X, \mathbb{Z})$.

Let $\Delta_{X} \subset X \times X$ be the diagonal in $X \times X$, and let $\left[\Delta_{3}\right]=\operatorname{id}_{H^{3}(X, \mathbb{Z})}$ be the component of $\left[\Delta_{X}\right]$ lying in $H^{3}(X, \mathbb{Z}) \otimes_{\mathbb{Z}} H^{3}(X, \mathbb{Z})$ in the Künneth decomposition of $H^{6}(X \times X, \mathbb{Z})$. Note that $\left[\Delta_{3}\right]$ is a Hodge class (i.e. it maps to $H^{3,3}(X)$ ), but it is not necessarily an analytic (algebraic) cycle class.

Let $\Delta_{S} \in S \times S$ be the diagonal in $S \times S$, and let $\left[\Delta_{2}\right]=\operatorname{id}_{H^{2}(S, \mathbb{Z})}$ be the component of $\left[\Delta_{S}\right]$ lying in $H^{2}(S, \mathbb{Z}) \otimes_{\mathbb{Z}} H^{2}(S, \mathbb{Z})$ in the Künneth decomposition of $H^{4}(S \times S, \mathbb{Z})$. Note that $\left[\Delta_{2}\right]$ is not only a Hodge class, but by a result of Murre ([Mur]) it is also analytic (algebraic).

We recall that for any complex analytic manifold $X$ there is a short exact sequence

$$
0 \rightarrow J^{2 p-1}(X) \rightarrow H_{\mathcal{D}}^{2 p}(X, \mathbb{Z}(p)) \rightarrow \operatorname{Hdg}^{2 p}(X) \rightarrow 0
$$

where

$$
J^{2 p-1}(X)=\frac{H^{2 p-1}(X, \mathbb{C})}{F^{p} H^{2 p-1}(X)+H^{2 p-1}(X, \mathbb{Z})}
$$

is the $p$ th Griffiths intermediate Jacobian of $X, H_{\mathcal{D}}$ denotes Deligne cohomology, which is defined as the hypercohomology of the complex of sheaves on $X$

$$
\mathbb{Z}(p)_{\mathcal{D}}: 0 \rightarrow \mathbb{Z}(p) \rightarrow \mathcal{O}_{X} \rightarrow \Omega_{X}^{1} \rightarrow \cdots \rightarrow \Omega_{X}^{p-1} \rightarrow 0,
$$

and $\operatorname{Hdg}^{2 p}(X)$ are the Hodge classes $\left\{\eta \in H^{2 p}(X, \mathbb{Z}): \alpha_{*} \eta \in H^{p, p}(X)\right\}$, with $\alpha_{*}: H^{2 p}(X, \mathbb{Z}) \rightarrow H^{2 p}(X, \mathbb{C})$ the natural map (see [EV] and [GMV] for descriptions).

For two classes $S$ in $X \times Y$ and $T$ in $Y \times Z$ (which may be cohomology classes in $H^{*}(X \times Y)$, Deligne cohomology classes in $H_{\mathcal{D}}^{*}(X \times Y)$, or cycle classes in $\mathrm{CH}^{p}(X \times Y)$ resp. ... $\left.(Y \times Z)\right)$ we define the composed correspondence $S \circ T:=p_{13 *}\left\{p_{12}^{*} S \cdot p_{23}^{*} T\right\}$ on $X \times Z$. Here "." denotes cup product, the product in Deligne cohomology and intersection product of cycles, respectively, and the $p_{i j}$ are the various projection maps from $X \times Y \times Z$ (see 
Chapter 16 in [Ful]). In the case of Deligne cohomology, we will denote the composition by "oD" ".

Now, since $\Gamma_{j}$ and $\Delta_{2}$ are both algebraic cycles, we can compose them to obtain $\Gamma_{j} \circ \Delta_{2} \subset S \times X$, with $\left[\Gamma_{j} \circ \Delta_{2}\right] \in H^{6}(S \times X, \mathbb{Z})$.

LEMma 3.1. $\left[\Delta_{3}\right] \circ\left[\Gamma_{j} \circ \Delta_{2}\right]=0$ on $S \times X$.

Proof. This is clear by reasons of cohomological degree.

For the cycle $\Gamma_{j} \circ \Delta_{2}$ we have the Deligne cycle class map

$$
\operatorname{cl}_{\mathcal{D}}: \mathrm{CH}^{3}(S \times X) \rightarrow H_{\mathcal{D}}^{6}(S \times X, \mathbb{Z}(3)), \quad \Gamma_{j} \circ \Delta_{2} \mapsto\left[\Gamma_{j} \circ \Delta_{2}\right]_{\mathcal{D}},
$$

which is compatible with the usual cycle class map cl, i.e. $\left[\Gamma_{j} \circ \Delta_{2}\right]_{\mathcal{D}}$ goes to $\left[\Gamma_{j} \circ \Delta_{2}\right]$ in the short exact sequence

$$
0 \rightarrow J^{5}(S \times X) \rightarrow H_{\mathcal{D}}^{6}(S \times X, \mathbb{Z}(3)) \rightarrow \operatorname{Hdg}^{6}(S \times X) \rightarrow 0 .
$$

Since we do not know whether $\left[\Delta_{3}\right] \in \operatorname{Hdg}^{6}(X \times X)$ is algebraic, we cannot use the Deligne cycle class map, but we can still lift it to a class $\left[\Delta_{3}\right]_{\mathcal{D}} \in$ $H_{\mathcal{D}}^{6}(X \times X, \mathbb{Z}(3))$ via the sequence

$$
0 \rightarrow J^{5}(X \times X) \rightarrow H_{\mathcal{D}}^{6}(X \times X, \mathbb{Z}(3)) \rightarrow \operatorname{Hdg}^{6}(X \times X) \rightarrow 0 .
$$

Then it is clear that $\left[\Delta_{3}\right]_{\mathcal{D}} \circ_{\mathcal{D}}\left[\Gamma_{j} \circ \Delta_{2}\right]_{\mathcal{D}} \in H_{\mathcal{D}}^{6}(S \times X, \mathbb{Z}(3))$ will map to $\left[\Delta_{3}\right] \circ\left[\Gamma_{j} \circ \Delta_{2}\right] \in \operatorname{Hdg}^{6}(S \times X)$, which we have just proven to be zero. Hence $\left[\Delta_{3}\right]_{\mathcal{D}}{ }^{\circ} \mathcal{D}\left[\Gamma_{j} \circ \Delta_{2}\right]_{\mathcal{D}}$ is in fact an element $\delta \in J^{5}(S \times X)=J\left(H^{5}(S \times X)\right)$. We introduce a convenient notation for the Jacobian of a (pure) Hodge structure of odd weight:

Definition 3.2. Let $\left(V_{\mathbb{Z}}, F^{\bullet}\right)$ be a pure Hodge structure of weight $2 k-1$, i.e. $V_{\mathbb{Z}}$ is a finitely generated free abelian $\mathbb{Z}$-module and there is a decomposition

$$
V_{\mathbb{C}}:=V_{\mathbb{Z}} \otimes_{\mathbb{Z}} \mathbb{C}=\bigoplus_{p+q=2 k-1} V^{p, q},
$$

where $V^{p, q}:=F^{p} V_{\mathbb{C}} \cap \overline{F^{q} V_{\mathbb{C}}}$ is defined via the Hodge filtration $F^{\bullet}$. Then we put

$$
J(V):=\frac{V_{\mathbb{C}}}{F^{k} V_{\mathbb{C}}+V_{\mathbb{Z}}}
$$

Now using the Künneth decomposition $H^{5}(S \times X)=\sum_{p=0}^{5} H^{p}(S) \otimes$ $H^{5-p}(X)$, we obtain a natural projection from $J\left(H^{5}(S \times X)\right)$ to

$$
J\left(H^{2}(S) \otimes H^{3}(X)\right) \cong H^{3}(X, \mathbb{Z}) \otimes_{\mathbb{Z}} H^{2}(S, \mathbb{Z}) \otimes_{\mathbb{Z}} \mathbb{R} / \mathbb{Z},
$$

and a further projection to

$$
J\left(\frac{H^{2}(S)}{j^{*} H^{2}(X)} \otimes \frac{H^{3}(X)}{j_{*} H^{1}(S)}\right) \cong \frac{H^{3}(X, \mathbb{Z})}{j_{*} H^{1}(S, \mathbb{Z})} \otimes_{\mathbb{Z}} \frac{H^{2}(S, \mathbb{Z})}{j^{*} H^{2}(X, \mathbb{Z})} \otimes_{\mathbb{Z}} \mathbb{R} / \mathbb{Z}
$$


(see Lemma 1.2 in [Gre1] for these isomorphisms). The projection $\delta^{\prime}$ of $\delta$ in this quotient can be seen as an element of $\frac{J^{3}(X)}{\operatorname{Pic}^{0}(S)} \otimes_{\mathbb{Z}} \frac{H^{2}(S, \mathbb{Z})}{j^{*} H^{2}(X, \mathbb{Z})}$.

LEMMA 3.3. $\delta^{\prime}$ is independent of the lifting $\left[\Delta_{3}\right]_{\mathcal{D}}$ of $\left[\Delta_{3}\right]$.

Proof. Suppose that we had chosen a different lifting $\left[\Delta_{3}\right]_{\mathcal{D}}+\nu, \nu \in$ $J^{5}(X \times X)$. We work with the projection maps from $S \times S \times X \times X$. We have

$$
\begin{aligned}
p_{34}^{*}\left(\left[\Delta_{3}\right]_{\mathcal{D}}\right. & +\nu) \cdot \mathcal{D} p_{23}^{*}\left[\Gamma_{j}\right]_{\mathcal{D}} \cdot \mathcal{D} p_{12}^{*}\left[\Delta_{2}\right]_{\mathcal{D}} \\
& =\left(p_{34}^{*}\left[\Delta_{3}\right]_{\mathcal{D}}+p_{34}^{*} \nu\right) \cdot \mathcal{D} p_{23}^{*}\left[\Gamma_{j}\right]_{\mathcal{D}} \cdot \mathcal{D} p_{12}^{*}\left[\Delta_{2}\right]_{\mathcal{D}} \\
& =p_{34}^{*}\left[\Delta_{3}\right]_{\mathcal{D}} \cdot \mathcal{D} p_{23}^{*}\left[\Gamma_{j}\right]_{\mathcal{D}} \cdot \mathcal{D} p_{12}^{*}\left[\Delta_{2}\right]_{\mathcal{D}}+p_{34}^{*} \nu \cdot p_{23}^{*}\left[\Gamma_{j}\right] \cdot p_{12}^{*}\left[\Delta_{2}\right]
\end{aligned}
$$

in $H_{\mathcal{D}}^{12}(S \times S \times X \times X, \mathbb{Z}(6))$, since the intermediate Jacobian is an ideal of square zero (see Proposition 7.10 in $[\mathrm{EV}]$ ), and where $\cdot$ denotes the action of a cohomology class on the intermediate Jacobian. Now via the Künneth formula we have the decomposition

$$
\nu \in J^{5}(X \times X)=J\left(H^{5}(X \times X)\right)=\bigoplus_{i=0}^{5} J\left(H^{i}(X) \otimes H^{5-i}(X)\right) .
$$

The class $\left[\Gamma_{j}\right]$ acts on $J\left(H^{5}(X \times X)\right)$ as $j^{*} \times \mathrm{id}_{X}$ (see Proposition 16.1.1 in [Ful] $)$, and the class $\left[\Delta_{2}\right]$ kills all the components other than $J\left(H^{2}(S) \otimes\right.$ $\left.H^{3}(X)\right)$. But anything in the image of $j^{*}: H^{2}(X) \rightarrow H^{2}(S)$ goes to zero in the quotient defined above, so we are done.

LEMMA 3.4. $\delta^{\prime}$ is independent of the choice of the representative $\Delta_{2} \in$ $\mathrm{CH}^{2}(S \times S)$ of $\left[\Delta_{2}\right]$.

Proof. The proof is similar to the previous one. Murre stresses in his paper [Mur, Section 5] that the choice of $\Delta_{2}$ as a cycle class is not canonical. What is clear, however, is that such a representative will be sent to $\left[\Delta_{2}\right] \in$ $H^{4}(S \times S, \mathbb{Z})$, and so again any two representatives will at most differ by an element $\mu \in J^{3}(S \times S)$ when mapped into the Deligne cohomology group $H_{\mathcal{D}}^{4}(S \times S, \mathbb{Z}(2))$.

The difference from the previous proof is that $\mu$ will be pushed forward via the graph of $j$, not pulled back, but this kind of element will be taken care of by the quotient $H^{3}(X) / j_{*} H^{1}(S)$. We obtain

$$
\begin{aligned}
p_{34}^{*}\left[\Delta_{3}\right]_{\mathcal{D}} \cdot \mathcal{D} & p_{23}^{*}\left[\Gamma_{j}\right]_{\mathcal{D}} \cdot \mathcal{D} p_{12}^{*}\left(\left[\Delta_{2}\right]_{\mathcal{D}}+\mu\right) \\
& =p_{34}^{*}\left[\Delta_{3}\right]_{\mathcal{D}} \cdot \mathcal{D} p_{23}^{*}\left[\Gamma_{j}\right]_{\mathcal{D}} \cdot \mathcal{D} p_{12}^{*}\left[\Delta_{2}\right]_{\mathcal{D}}+p_{34}^{*}\left[\Delta_{3}\right] \cdot p_{23}^{*}\left[\Gamma_{j}\right] \cdot p_{12}^{*} \mu
\end{aligned}
$$

in $H_{\mathcal{D}}^{12}(S \times S \times X \times X, \mathbb{Z}(6))$. We have the decomposition

$$
\mu \in J\left(H^{3}(S \times S)\right)=\bigoplus_{i=0}^{3} J\left(H^{i}(S) \otimes H^{3-i}(S)\right) .
$$


Now $\left[\Gamma_{j}\right]$ acts on $J^{3}(S \times S)$ as $\operatorname{id}_{S} \times j_{*}$, and this time $\left[\Delta_{3}\right]$ only leaves $J\left(H^{2}(S) \otimes H^{3}(X)\right)$, which completes the proof.

We have to work a little more to obtain our final invariant $d_{X, S}$.

Definition 3.5. Let $H^{2}(S, \mathbb{Z})_{\text {new }}=\operatorname{ker}\left(j_{*}: H^{2}(S, \mathbb{Z}) \rightarrow H^{4}(X, \mathbb{Z})\right)$.

Then we have $H^{2}(S, \mathbb{Z})_{\text {new }}^{*}=H^{2}(S, \mathbb{Z}) / j^{*} H^{2}(X, \mathbb{Z})$. In order to contract $d_{X, S}$ and $e_{S, C} \cdot f_{C, Z}$ to $d_{X, S} \cdot e_{S, C} \cdot f_{C, Z}$, we quotient $J^{3}(X)$ by the image of the Abel-Jacobi map $\phi_{X}: \mathcal{Z}^{2}(X)_{\text {hom }} \rightarrow J^{3}(X)$, where $\mathcal{Z}^{2}(X)_{\text {hom }}$ denotes the group of codimension two algebraic cycles of $X$ which are homologically equivalent to zero.

Definition 3.6. $J^{3}(X)_{\mathrm{AJ}}:=J^{3}(X) / \operatorname{im}\left(\phi_{X}\right)$.

Note that $\operatorname{im}\left(\phi_{X}\right) \supseteq j_{*} H^{1}(S, \mathbb{Z}) \otimes_{\mathbb{Z}} \mathbb{R} / \mathbb{Z}=j_{*} \operatorname{Pic}^{0}(S)$.

Definition 3.7. We define $d_{X, S}$ to be the projection of $\delta^{\prime}$ in

$$
J^{3}(X)_{\mathrm{AJ}} \otimes_{\mathbb{Z}} H^{2}(S, \mathbb{Z})_{\text {new }}^{*} .
$$

We now specify our choice of $C$ and $S$ : since by assumption $Z_{0}$ lies in the kernel of the map $\psi_{2}^{3}$, we know that there exists a surface $S$, a curve $C$ and a lifting $Z$ of $Z_{0}$ such that the invariant $e_{S, C} \cdot f_{C, Z} \in H^{2}(S, \mathbb{Z})_{\mathrm{tr}}^{*} \otimes_{\mathbb{Z}} \mathbb{R} / \mathbb{Z}^{\otimes 2}$ belongs to $H^{2}(S, \mathbb{Z})_{\mathrm{tr}, \text { new }}^{*} \otimes_{\mathbb{Z}} \mathbb{R} / \mathbb{Z}^{\otimes 2}$, where

$$
H^{2}(S, \mathbb{Z})_{\mathrm{tr}, \text { new }}^{*}:=\operatorname{ker}\left(j_{*}: H^{2}(S, \mathbb{Z})_{\mathrm{tr}}^{*}=\frac{H^{2}(S, \mathbb{Z})}{\mathrm{NS}(S)} \rightarrow \frac{H^{4}(X, \mathbb{Z})}{j_{*} \mathrm{NS}(S)}\right) .
$$

Lift $e_{S, C} \cdot f_{C, Z}$ to an element in $H^{2}(S, \mathbb{Z})_{\text {new }} \otimes_{\mathbb{Z}} \mathbb{R} / \mathbb{Z}^{\otimes 2}$. This lifting will depend upon the choice of an element $\alpha \in \operatorname{ker}\left(j_{*}: \operatorname{NS}(S) \rightarrow H^{4}(X, \mathbb{Z})\right)$. In order to have a well defined contraction between $d_{X, S}$ and $e_{S, C} \cdot f_{C, Z}$ via the pairing $H^{2}(S, \mathbb{Z})_{\text {new }}^{*} \otimes_{\mathbb{Z}} H^{2}(S, \mathbb{Z})_{\text {new }} \rightarrow \mathbb{Z}$, we must prove the following:

LEMma 3.8 .

$$
d_{X, S} \cdot e_{S, C} \cdot f_{C, Z} \in J^{3}(X)_{\mathrm{AJ}} \otimes_{\mathbb{Z}} \mathbb{R} / \mathbb{Z}^{\otimes 2}
$$

is independent of the element $\alpha$ chosen in the lifting.

Proof. We can view

$$
\delta^{\prime} \in \frac{J^{3}(X)}{j_{*} \operatorname{Pic}^{0}(S)} \otimes_{\mathbb{Z}} \frac{H^{2}(S, \mathbb{Z})}{j^{*} H^{2}(X, \mathbb{Z})}
$$

as an element

$$
\delta^{\prime} \in \operatorname{Hom}\left(H^{2}(S, \mathbb{Z}) \cap \operatorname{ker} j_{*}, \frac{J^{3}(X)}{j_{*} \operatorname{Pic}^{0}(S)}\right) .
$$

We now prove

Lemma 3.9. The restriction of $\delta^{\prime}$ (the coboundary map in the diagram below) to $\operatorname{NS}(S) \cap \operatorname{ker} j_{*}$ is equal to the composition

$$
\phi_{X} \circ j_{*}: \operatorname{NS}(S) \cap \operatorname{ker} j_{*} \rightarrow \mathcal{Z}(X)_{\text {hom }}^{2} / j_{*} \operatorname{Pic}^{0}(S) \rightarrow J^{3}(X) / j_{*} \operatorname{Pic}^{0}(S) .
$$


Proof of Lemma 3.9. Consider the action of

$$
\delta=\left[\Delta_{3}\right]_{\mathcal{D}} \circ_{\mathcal{D}}\left[\Gamma_{j}\right]_{\mathcal{D}} \circ_{\mathcal{D}}\left[\Delta_{2}\right]_{\mathcal{D}}
$$

on a class $\alpha \in \operatorname{NS}(S) \cap \operatorname{ker} j_{*} \subseteq \operatorname{NS}(S)=\operatorname{Hdg}^{2}(S)$, which we can lift to an element $\widetilde{\alpha} \in H_{\mathcal{D}}^{2}(S, \mathbb{Z}(1)) \cong \operatorname{Pic}(S)$ :

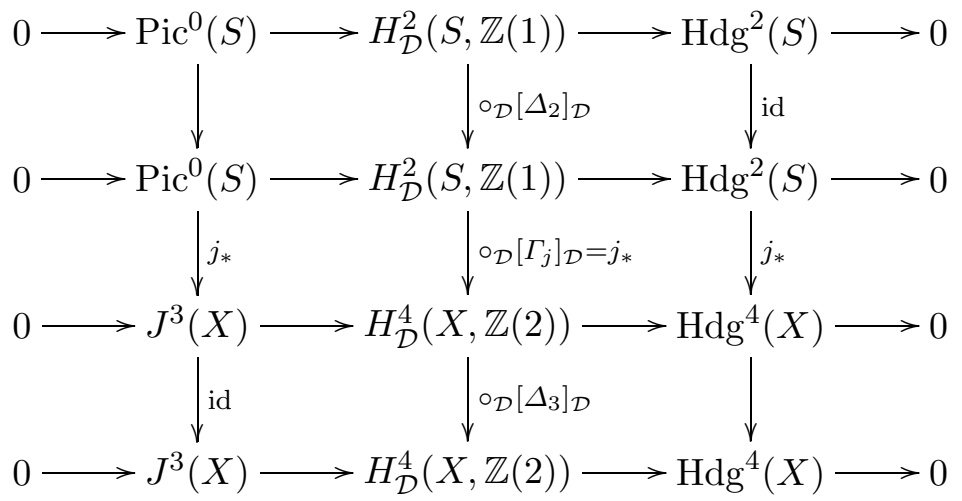

Since $j_{*}(\alpha)=0 \in \operatorname{Hdg}^{4}(X), j_{*}(\widetilde{\alpha})$ gives an element of $J^{3}(X)$, which is precisely the Abel-Jacobi image $\phi_{X}\left(j_{*}(\alpha)\right.$ ) (see Proposition 1 in [EZ], for example), and which is well defined up to an element in $j_{*} \operatorname{Pic}^{0}(S)$.

But since we have quotiented by the image of the Abel-Jacobi map $\phi_{X}$ in the definition of $d_{X, S}$, we see that the choice of $\alpha$ makes no difference in our construction; this completes the proof of Lemma 3.8.

Now we can define the higher Jacobian $J_{3}^{3}(X)$ and the corresponding map $\psi_{3}^{3}$ for 0 -cycles on $X$ :

Definition 3.10. Let $U_{3}^{3}(X)$ be the group generated by all $d_{X, S} \cdot e_{S, C}$. $f_{C, Z}$ for which $(j \circ i)_{*}(Z)=0$ as a 0 -cycle of $X$, let

$$
J_{3}^{3}(X)=\frac{J^{3}(X)_{\mathrm{AJ}} \otimes_{\mathbb{Z}} \mathbb{R} / \mathbb{Z}^{\otimes 2}}{U_{3}^{3}(X)}
$$

and define the map

$$
\psi_{3}^{3}: \operatorname{ker}\left(\psi_{2}^{3}\right) \rightarrow J_{3}^{3}(X), \quad Z_{0} \mapsto\left[d_{X, S} \cdot e_{S, C} \cdot f_{C, Z}\right] .
$$

What remains to be shown in order to have a well defined map is this:

Lemma 3.11. $\psi_{3}^{3}\left(Z_{0}\right)$ is independent of the choice of the surface $S$.

Proof. The proof is like the one of Lemma 2.3: suppose again that $Z_{1} \rightarrow$ $C_{1} \rightarrow S_{1}$ and $Z_{2} \rightarrow C_{2} \rightarrow S_{2}$ are both 0 -cycles mapping to $Z_{0}$ on $X$. Then we immediately have

$$
\begin{aligned}
d_{X, S_{1}} \cdot e_{S_{1}, C_{1}} \cdot f_{C_{1}, Z_{1}}-d_{X, S_{2}} \cdot e_{S_{2}, C_{2}} \cdot f_{C_{2}, Z_{2}} & \\
= & d_{X, S_{1} \sqcup S_{2}} \cdot e_{S_{1} \sqcup S_{2}, C_{1} \sqcup C_{2}} \cdot f_{C_{1} \sqcup C_{2}, Z_{1}-Z_{2}} \in U_{3}^{3}(X) .
\end{aligned}
$$


Finally, we mention that - for exactly the same reason as $\psi_{2}^{3}$ in Section 2 - the map $\psi_{3}^{3}$ factors through rational equivalence, and so indeed defines a map from $\operatorname{ker}\left(\psi_{2}^{3}\right) \subset \mathrm{CH}_{0}(X)$ to $J_{3}^{3}(X)$.

4. A formula for the pull-back of holomorphic 3-forms. In this section we prove that Mumford's pull-back of a holomorphic 3-form on $X$ to a variety parametrizing 0 -cycles on $X$ can be computed with our three AbelJacobi invariants - this is Theorem 4.5. We change our point of view: instead of considering fixed 0 -cycles, curves and surfaces, we now let them vary in a family parametrized by an open complex ball $B$. This allows us to consider the cohomology groups used before as local systems and the Abel-Jacobi invariants as maps from the base $B$ which we can then differentiate using the Gauss-Manin connection. First we give a definition of Green's pull-back of a holomorphic form in order to state the theorem. Then we prove the theorem. The homomorphisms $d \delta_{\mathbb{C}}^{\prime}, d e_{\mathbb{C}}^{\prime}$ and $d f_{\mathbb{C}}$ are derived from the Abel-Jacobi invariants $\delta_{X, S}^{\prime}, e_{S, C}$ and $f_{C, Z}$ of the previous section, and at each step we describe the action of these maps geometrically (see Propositions 4.6, 4.9 and 4.12). Combining these three steps finally leads to our formula.

4.1. Statement of the theorem. We let our curves and surfaces vary in families parametrized by the same open complex ball $B$. Consider the following situation: Let $\mathcal{C}$ and $\mathcal{S}$ be smooth complex varieties with proper submersive holomorphic maps $\pi$ and $\varrho$ to $B$ of relative dimensions 1 and 2, respectively. Let $i: \mathcal{C} \rightarrow \mathcal{S}$ and $j: \mathcal{S} \rightarrow X \times B$ be analytic morphisms making both squares in the diagram below commute:

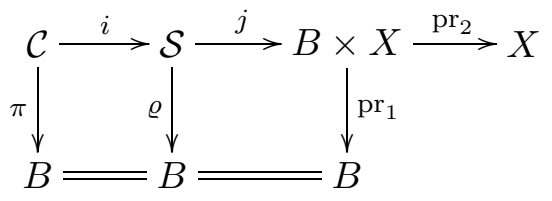

Let $s_{1}, \ldots, s_{N}$ be holomorphic sections of $\pi: \mathcal{C} \rightarrow B$, and let $m_{1}, \ldots, m_{N}$ be integers such that the 0 -cycle

$$
Z_{b}:=\sum_{i=0}^{N} m_{i} s_{i}(b)
$$

satisfies the following conditions for all $b \in B$ :

1. It is of degree 0 on each component of the curve $C_{b}$,

2. $i_{b *} Z_{b}$ is Abel-Jacobi equivalent to 0 in $S_{b}$, and

3. $j_{b *}\left(e_{b} \cdot f_{b}\right)=0$ in $H^{4}(X, \mathbb{Z}) / j_{b *} \mathrm{NS}\left(S_{b}\right) \otimes_{\mathbb{Z}}(\mathbb{R} / \mathbb{Z})^{\otimes 2}$,

where $e_{b}=e_{S_{b}, C_{b}}, f_{b}=f_{C_{b}, Z_{b}}$, and $e_{b} \cdot f_{b} \in H^{2}\left(S_{b}, \mathbb{Z}\right)_{\mathrm{tr}}^{*} \otimes_{\mathbb{Z}}(\mathbb{R} / \mathbb{Z})^{\otimes 2}$ is Green's contraction introduced in the previous section. 
We would like to differentiate the invariants $\left(e_{b}\right)_{b \in B}$ with respect to the parameters. However, since the groups $H^{2}\left(S_{b}, \mathbb{Z}\right)_{\text {tr }}$ do not form a local system (the Néron-Severi group may jump), we cannot do that.

Instead, let $e_{b}^{\prime}=e_{S_{b}, C_{b}}^{\prime}$ be the extension class of the short exact sequence of mixed Hodge structures

$$
0 \rightarrow \frac{H^{1}\left(C_{b}\right)}{i_{b}^{*} H^{1}\left(S_{b}\right)} \rightarrow H^{2}\left(S_{b}, C_{b}\right) \rightarrow H^{2}\left(S_{b}\right)_{C_{b}} \rightarrow 0,
$$

where $H^{2}\left(S_{b}\right)_{C_{b}}:=\operatorname{ker}\left(i_{b}^{*}: H^{2}\left(S_{b}\right) \rightarrow H^{2}\left(C_{b}\right)\right)$. Identifying the intermediate Jacobian in which $e_{b}^{\prime}$ lies with its underlying real torus, we have

$$
\begin{aligned}
e_{b}^{\prime} \in & \frac{H^{1}\left(C_{b}, \mathbb{Z}\right)}{i_{b}^{*} H^{1}\left(S_{b}, \mathbb{Z}\right)} \otimes_{\mathbb{Z}}\left(H^{2}\left(S_{b}, \mathbb{Z}\right)_{C_{b}}\right)^{*} \otimes_{\mathbb{Z}} \mathbb{R} / \mathbb{Z} \\
= & \frac{H^{1}\left(C_{b}, \mathbb{Z}\right)}{i_{b}^{*} H^{1}\left(S_{b}, \mathbb{Z}\right)} \otimes_{\mathbb{Z}} \frac{H^{2}\left(S_{b}, \mathbb{Z}\right)}{\left\langle C_{b, r}\right\rangle} \otimes_{\mathbb{Z}} \mathbb{R} / \mathbb{Z},
\end{aligned}
$$

where the $C_{b, r}$ are the components of the curve $C_{b}$. We know that the previous invariant $e_{b}$ is obtained by projecting $e_{b}^{\prime}$ via the quotient map

$$
\frac{H^{2}\left(S_{b}, \mathbb{Z}\right)}{\left\langle C_{b, r}\right\rangle} \rightarrow \frac{H^{2}\left(S_{b}, \mathbb{Z}\right)}{\left\langle\mathrm{NS}\left(S_{b}\right)\right\rangle}=H^{2}(S, \mathbb{Z})_{\mathrm{tr}}^{*}
$$

Now since the first two quotients in the last expression above are locally constant, and $B$ is simply connected, we have natural identifications

$$
\frac{H^{1}\left(C_{b}, \mathbb{Z}\right)}{i_{b}^{*} H^{1}\left(S_{b}, \mathbb{Z}\right)} \cong \frac{H^{1}\left(C_{0}, \mathbb{Z}\right)}{i_{b}^{*} H^{1}\left(S_{0}, \mathbb{Z}\right)} \quad \text { and } \quad \frac{H^{2}\left(S_{b}, \mathbb{Z}\right)}{\left\langle C_{b, r}\right\rangle} \cong \frac{H^{2}\left(S_{0}, \mathbb{Z}\right)}{\left\langle C_{0, r}\right\rangle}
$$

Next, the Abel-Jacobi invariant

$$
f_{b}=\operatorname{alb}\left(Z_{b}\right) \in J\left(C_{b}\right) \cong H^{1}\left(C_{b}, \mathbb{Z}\right) \otimes_{\mathbb{Z}} \mathbb{R} / \mathbb{Z}
$$

belongs to $H^{1}\left(C_{b}, \mathbb{Z}\right)_{\text {new }} \otimes_{\mathbb{Z}} \mathbb{R} / \mathbb{Z}$ by our second assumption, where $H^{1}\left(C_{b}, \mathbb{Z}\right)_{\text {new }}:=\operatorname{ker}\left(i_{b *}: H^{1}\left(C_{b}, \mathbb{Z}\right) \rightarrow H^{3}\left(S_{b}, \mathbb{Z}\right)\right)$. The groups $H^{1}\left(C_{b}, \mathbb{Z}\right)_{\text {new }}$ form a local system, and as above we identify canonically $H^{1}\left(C_{b}, \mathbb{Z}\right)_{\text {new }} \cong$ $H^{1}\left(C_{0}, \mathbb{Z}\right)_{\text {new }}$. So now we can view $\left(e_{b}^{\prime}\right)_{b \in B}$ and $\left(f_{b}\right)_{b \in B}$ as maps

$$
e^{\prime}: B \rightarrow \frac{H^{1}\left(C_{0}, \mathbb{Z}\right)}{i_{b}^{*} H^{1}\left(S_{0}, \mathbb{Z}\right)} \otimes_{\mathbb{Z}} \frac{H^{2}\left(S_{0}, \mathbb{Z}\right)}{\left\langle C_{0, r}\right\rangle} \otimes_{\mathbb{Z}} \mathbb{R} / \mathbb{Z}, \quad b \mapsto e_{b}^{\prime},
$$

and

$$
f: B \rightarrow H^{1}\left(C_{0}, \mathbb{Z}\right)_{\text {new }} \otimes_{\mathbb{Z}} \mathbb{R} / \mathbb{Z}, \quad b \mapsto f_{b},
$$

which are differentiable - in fact they are even real-analytic, since we know by Griffiths' results that the Abel-Jacobi invariants vary holomorphically in the family. Differentiating these two functions gives maps

$$
d e^{\prime}: T_{B}^{\mathbb{R}} \rightarrow \frac{H^{1}\left(C_{0}, \mathbb{Z}\right)}{i_{0}^{*} H^{1}\left(S_{0}, \mathbb{Z}\right)} \otimes_{\mathbb{Z}} \frac{H^{2}\left(S_{0}, \mathbb{Z}\right)}{\left\langle C_{0, r}\right\rangle} \otimes_{\mathbb{Z}} \mathbb{R}
$$


and

$$
d f: T_{B}^{\mathbb{R}} \rightarrow H^{1}\left(C_{0}, \mathbb{Z}\right)_{\text {new }} \otimes_{\mathbb{Z}} \mathbb{R}
$$

and by adjunction real differential forms

$$
d e^{\prime} \in \frac{H^{1}\left(C_{0}, \mathbb{Z}\right)}{i_{0}^{*} H^{1}\left(S_{0}, \mathbb{Z}\right)} \otimes_{\mathbb{Z}} \frac{H^{2}\left(S_{0}, \mathbb{Z}\right)}{\left\langle C_{0, r}\right\rangle} \otimes_{\mathbb{Z}} \Omega_{B}^{\mathbb{R}}
$$

and

$$
d f \in H^{1}\left(C_{0}, \mathbb{Z}\right)_{\text {new }} \otimes_{\mathbb{Z}} \Omega_{B}^{\mathbb{R}} .
$$

REMARK 4.1. Instead of trivializing the local systems and differentiating the functions $b \mapsto e_{b}^{\prime}, b \mapsto f_{b}$, we could have introduced the Gauss-Manin connections $\nabla$ on the local systems considered, and applied them to our sections $e_{b}^{\prime}$ and $f_{b}$. The two points of view are equivalent and both will be adopted in what follows. The advantage of the second point of view is that one does not need to distinguish a point 0 .

Finally, contracting via the natural pairing between $H^{1}\left(C_{0}, \mathbb{Z}\right)_{\text {new }}$ and $H^{1}\left(C_{0}, \mathbb{Z}\right) / i_{0}^{*} H^{1}\left(S_{0}, \mathbb{Z}\right)=H^{1}\left(C_{0}, \mathbb{Z}\right)_{\text {new }}^{*}$, and using wedge product for 1 forms, we obtain a real 2 -form

$$
d e^{\prime} \wedge d f \in \bigwedge^{2} \Omega_{B}^{\mathbb{R}} \otimes_{\mathbb{Z}} H^{2}\left(S_{0}, \mathbb{Z}\right) /\left\langle C_{0, r}\right\rangle .
$$

Now we do the following: Firstly, by the duality

$$
H^{2}\left(S_{0}, \mathbb{Z}\right) /\left\langle C_{0, r}\right\rangle \cong\left(H^{2}\left(S_{b}, \mathbb{Z}\right)_{C_{b}}\right)^{*}
$$

this 2-form can be viewed as an element

$$
d e^{\prime} \wedge d f \in \operatorname{Hom}_{\mathbb{Z}}\left(H^{2}\left(S_{b}, \mathbb{Z}\right)_{C_{b}}, \bigwedge^{2} \Omega_{B}^{\mathbb{R}}\right),
$$

which gives, by $\mathbb{C}$-linear extension, an element

$$
d e^{\prime} \wedge d f_{\mathbb{C}} \in \operatorname{Hom}_{\mathbb{C}}\left(H^{2}\left(S_{b}, \mathbb{C}\right)_{C_{b}}, \wedge^{2} \Omega_{B}^{\mathbb{C}}\right) .
$$

Secondly, it follows from our third hypothesis that the 2-form (7) actually takes values in

$$
\bigwedge^{2} \Omega_{B}^{\mathbb{R}} \otimes_{\mathbb{Z}} \operatorname{ker}\left(j_{0 *}: \frac{H^{2}\left(S_{0}, \mathbb{Z}\right)}{\left\langle C_{0, r}\right\rangle} \rightarrow \frac{H^{4}(X, \mathbb{Z})}{j_{0 *}\left(\mathrm{NS}\left(S_{0}\right)\right)}\right),
$$

so that our homomorphism $d e^{\prime} \wedge d f_{\mathbb{C}}$ in fact vanishes on

$$
j_{0}^{*}\left(H^{2}(X, \mathbb{C}) \cap\left(j_{0 *}\left(\mathrm{NS}\left(S_{0}\right)\right)^{\perp}\right)\right) .
$$

In particular, it will vanish on $j_{0}^{*} H^{2,0}(X)$, which will be crucial for a pairing to come later, since we will be dealing with a complex 2 -form of type $(2,0)$ on the surface $S_{0}$ which is only defined up to the pull-back of a $(2,0)$-form on $X$.

We will similarly need to differentiate the third Abel-Jacobi invariant: Recall that

$$
\delta_{b}^{\prime} \in \frac{H^{2}\left(S_{b}, \mathbb{Z}\right)}{j_{b}^{*} H^{2}(X, \mathbb{Z})} \otimes_{\mathbb{Z}}\left(H^{3}(X, \mathbb{Z})_{S_{b}}\right)^{*} \otimes_{\mathbb{Z}} \mathbb{R} / \mathbb{Z}
$$


where

$$
H^{3}(X, \mathbb{Z})_{S_{b}}:=\operatorname{ker}\left(j_{b}^{*}: H^{3}(X, \mathbb{Z}) \rightarrow H^{3}\left(S_{b}, \mathbb{Z}\right)\right) .
$$

The groups $H^{2}\left(S_{b}, \mathbb{Z}\right) / j_{b}^{*} H^{2}(X, \mathbb{Z})$ and $H^{3}(X, \mathbb{Z})_{S_{b}}$ form a local system, hence we have natural identifications

$$
\frac{H^{2}\left(S_{b}, \mathbb{Z}\right)}{j_{b}^{*} H^{2}(X, \mathbb{Z})} \cong \frac{H^{2}\left(S_{0}, \mathbb{Z}\right)}{j_{0}^{*} H^{2}(X, \mathbb{Z})} \quad \text { and } \quad H^{3}(X, \mathbb{Z})_{S_{b}} \cong H^{3}(X, \mathbb{Z})_{S_{0}}
$$

This allows us to view $\delta^{\prime}$ as a map

$$
B \rightarrow \frac{H^{2}\left(S_{0}, \mathbb{Z}\right)}{j_{0}^{*} H^{2}(X, \mathbb{Z})} \otimes_{\mathbb{Z}}\left(H^{3}(X, \mathbb{Z})_{S_{0}}\right)^{*} \otimes_{\mathbb{Z}} \mathbb{R} / \mathbb{Z}, \quad b \mapsto \delta_{b}^{\prime},
$$

which again by Griffiths' results is differentiable and even real-analytic. Its differential is a map

$$
d \delta^{\prime}: T_{B}^{\mathbb{R}} \rightarrow \frac{H^{2}\left(S_{0}, \mathbb{Z}\right)}{j_{0}^{*} H^{2}(X, \mathbb{Z})} \otimes_{\mathbb{Z}}\left(H^{3}(X, \mathbb{Z})_{S_{0}}\right)^{*} \otimes_{\mathbb{Z}} \mathbb{R}
$$

or a real 1-form

$$
d \delta^{\prime} \in \frac{H^{2}\left(S_{0}, \mathbb{Z}\right)}{j_{0}^{*} H^{2}(X, \mathbb{Z})} \otimes_{\mathbb{Z}}\left(H^{3}(X, \mathbb{Z})_{S_{0}}\right)^{*} \otimes_{\mathbb{Z}} \Omega_{B}^{\mathbb{R}} .
$$

This 1-form in turn can be viewed as an element

$$
d \delta^{\prime} \in \operatorname{Hom}_{\mathbb{Z}}\left(H^{3}(X, \mathbb{Z})_{S_{0}}, \frac{H^{2}\left(S_{0}, \mathbb{Z}\right)}{j_{0}^{*} H^{2}(X, \mathbb{Z})} \otimes_{\mathbb{Z}} \Omega_{B}^{\mathbb{R}}\right),
$$

and again, by $\mathbb{C}$-linear extension, as

$$
d \delta_{\mathbb{C}}^{\prime} \in \operatorname{Hom}_{\mathbb{C}}\left(H^{3}(X, \mathbb{C})_{S_{0}}, \frac{H^{2}\left(S_{0}, \mathbb{C}\right)}{j_{0}^{*} H^{2}(X, \mathbb{C})} \otimes_{\mathbb{C}} \Omega_{B}^{\mathbb{C}}\right) .
$$

The main result of this section concerns pull-backs to $B$ of global holomorphic 3 -forms on $X$. Before we can state it, we need a lemma. Note that simply by reason of type, a $(3,0)$-form $\omega$ on $X$ will vanish when pulled back to the surface $S_{b}$, so that its class $[\omega]$ belongs to $H^{3}(X, \mathbb{C})_{S_{b}}$. Hence we can apply our homomorphism $d \delta_{\mathbb{C}}^{\prime}$ to it, and the following statement makes sense:

Lemma 4.2. Let $\omega$ be a form of type $(3,0)$ on $X$. Then at $0 \in B$,

$$
d \delta_{\mathbb{C}}^{\prime}([\omega]) \in \frac{H^{2,0}\left(S_{0}\right)}{j_{0}^{*} H^{2,0}(X)} \otimes_{\mathbb{C}} \Omega_{B, 0}^{1,0} \subset \frac{H^{2}\left(S_{0}, \mathbb{C}\right)}{j_{0}^{*} H^{2}(X, \mathbb{C})} \otimes_{\mathbb{C}} \Omega_{B, 0}^{\mathbb{C}} .
$$

This lemma is an immediate consequence of Proposition 4.6, which gives an explicit description of the map $d \delta_{\mathbb{C}}^{\prime}$ and will be proved later in this section. Assuming it and using the remarks made after the definition of

$$
d e^{\prime} \wedge d f_{\mathbb{C}} \in \operatorname{Hom}_{\mathbb{C}}\left(H^{2}\left(S_{0}, \mathbb{C}\right)_{C_{0}}, \wedge^{2} \Omega_{B}^{\mathbb{C}}\right),
$$


it follows that $d e^{\prime} \wedge d f_{\mathbb{C}}\left(d \delta_{\mathbb{C}}^{\prime}([\omega])\right) \in \Omega_{B, 0, \mathbb{C}}^{3}$ is well defined. Here we use contraction and wedge products of forms. Notice that, as mentioned in Remark 4.1, Lemma 4.2 holds true at any point (with 0 replaced by $b \in B$ everywhere), so that the construction in fact works over $B$ and thus provides a 3 -form on $B$.

Definition 4.3. We call

$$
d \delta^{\prime} \wedge d e^{\prime} \wedge d f([\omega]):=d e^{\prime} \wedge d f_{\mathbb{C}}\left(d \delta_{\mathbb{C}}^{\prime}([\omega])\right)
$$

Green's pull-back of the holomorphic 3-form $\omega$ on $X$ to $B$.

Next, following Mumford [Mum], another way to obtain a 3-form on $B$ from one on $X$ is this: Define the maps

$$
\theta_{i}=\operatorname{pr}_{2} \circ j \circ i \circ s_{i}: B \rightarrow X, \quad i=1, \ldots, N,
$$

and recall that $m_{1}, \ldots, m_{N}$ are the coefficients of the 0 -cycles

$$
Z_{b}=\sum_{i=0}^{N} m_{i} s_{i}(b), \quad b \in B
$$

on the curves $C_{b}$.

DEFINITION 4.4. We call

$$
M^{*}(\omega):=\sum_{i=0}^{N} m_{i} \theta_{i}^{*}(\omega)
$$

Mumford's pull-back of the holomorphic 3-form $\omega$ to the variety $B$ parametrizing 0-cycles on $X$.

Now we can finally state our main result of this section:

THEOREM 4.5. Let $\omega$ be a holomorphic 3-form on $X$. Then $d \delta^{\prime} \wedge d e^{\prime} \wedge$ $d f([\omega])$ is holomorphic and equal (up to sign) to $M^{*}(\omega)$, i.e. Green's and Mumford's pull-backs of $\omega$ to $B$ are the same (up to sign).

4.2. The proof of Theorem 4.5. For the proof, we shall use the following alternative description of the Abel-Jacobi invariants $e^{\prime}$ and $\delta^{\prime}$.

Let $\phi: X \rightarrow Y$ be a morphism of smooth projective varieties. There is a mixed Hodge structure on the relative cohomology groups $H^{*}(Y, X, \mathbb{Z})$ which fit in the long exact sequence

$$
\cdots \rightarrow H^{*}(Y, X) \rightarrow H^{*}(Y) \rightarrow H^{*}(X) \rightarrow H^{*+1}(Y, X) \rightarrow \cdots
$$

This sequence splits into short exact sequences of mixed Hodge structures

$$
0 \rightarrow \frac{H^{*-1}(X)}{\phi^{*} H^{*-1}(Y)} \rightarrow H^{*}(Y, X) \rightarrow H^{*}(Y)_{X} \rightarrow 0,
$$

where as usual $H^{*}(Y)_{X}:=\operatorname{ker}\left(H^{*}(Y) \rightarrow H^{*}(X)\right)$. It is known by results of Carlson [Car1, Car2] that this exact sequence of mixed Hodge structures is 
described by its extension class

$$
e_{Y, X} \in J\left(\operatorname{Hom}\left(H^{*}(Y)_{X}, \frac{H^{*-1}(X)}{\phi^{*} H^{*-1}(Y)}\right)\right) .
$$

It turns out that these extension classes are exactly the Abel-Jacobi invariants associated to the cycle $\Gamma_{\phi}=\operatorname{graph}(\phi)$ of $X \times Y$. From now on we will use this fact freely.

STEP 1: The action of $d \delta_{\mathbb{C}}^{\prime}$. Let $\omega$ be a holomorphic 3-form on $X$, let $\Omega_{B}^{k}$ and $\Omega_{\mathcal{S}}^{k}$ denote the sheaves of holomorphic $k$-forms on $B$ and $\mathcal{S}$, respectively. Define $\mathcal{H}_{\mathcal{S}}^{2}=\mathbf{R}^{2} \varrho_{*} \mathbb{C} \otimes \mathcal{O}_{B}$, and let $\mathcal{H}_{X}^{2}$ be the trivial bundle over $B$ with fiber $H^{2}(X, \mathbb{C})$. Consider the following sequence of sheaves on $\mathcal{S}$, defining $K$ :

$$
0 \rightarrow K \rightarrow \Omega_{\mathcal{S}}^{3} \stackrel{p}{\rightarrow} \Omega_{\mathcal{S} / B}^{2} \otimes \varrho^{*} \Omega_{B}^{1} \rightarrow 0
$$

We project the section $j^{*} \omega$ of $\Omega_{\mathcal{S}}^{3}$ to the section $p\left(j^{*} \omega\right)$ of $\Omega_{\mathcal{S} / B}^{2} \otimes \varrho^{*} \Omega_{B}^{1}$, further via $\varrho_{*}$ to a section still denoted by $p\left(j^{*} \omega\right)$ of

$$
\varrho_{*} \Omega_{\mathcal{S} / B}^{2} \otimes \varrho_{*} \varrho^{*} \Omega_{B}^{1}=\mathcal{H}_{\mathcal{S}}^{2,0} \otimes \Omega_{B}^{1} \subseteq \mathcal{H}_{\mathcal{S}}^{2} \otimes \Omega_{B}^{1},
$$

and finally via the projection $\mathcal{H}_{\mathcal{S}}^{2,0} \rightarrow \mathcal{H}_{\mathcal{S}}^{2,0} / j^{*} \mathcal{H}_{X}^{2,0}$ to a section

$$
\alpha(\omega) \in \frac{\mathcal{H}_{\mathcal{S}}^{2,0}}{j^{*} \mathcal{H}_{X}^{2,0}} \otimes \Omega_{B}^{1}
$$

Here $\mathcal{H}_{\mathcal{S}}^{2,0} \subset \mathcal{H}_{\mathcal{S}}^{2}$ and $\mathcal{H}_{X}^{2,0} \subset \mathcal{H}_{X}^{2}$ are the Hodge bundles with fibers $H^{2,0}\left(S_{b}\right)$ $\cong H^{0}\left(\Omega_{S_{b}}^{2}\right) \subset H^{2}\left(S_{b}, \mathbb{C}\right)$ and $H^{2,0}(X) \subset H^{2}(X, \mathbb{C})$, respectively.

Proposition 4.6. Let $\omega$ be a holomorphic 3 -form on $X$. Then for any $b \in B$ we have the equality

$$
d \delta_{\mathbb{C}}^{\prime}([\omega])_{b}=\alpha(\omega)_{b}
$$

via the inclusion

$$
\frac{H^{2,0}\left(S_{b}, \mathbb{C}\right)}{j_{b}^{*} H^{2,0}(X, \mathbb{C})} \otimes_{\mathbb{C}} \Omega_{B, b}^{1} \subseteq \frac{H^{2}\left(S_{b}, \mathbb{C}\right)}{j_{b}^{*} H^{2}(X, \mathbb{C})} \otimes_{\mathbb{C}} \Omega_{B, b}^{\mathbb{C}} \cong \frac{H^{2}\left(S_{0}, \mathbb{C}\right)}{j_{0}^{*} H^{2}(X, \mathbb{C})} \otimes_{\mathbb{C}} \Omega_{B, b}^{\mathbb{C}}
$$

Proof. By Carlson's description of $\delta_{X, S_{b}}^{\prime}$ as an extension class it is the class of $\sigma_{F}-\sigma_{\mathbb{Z}}$ in the quotient

$$
\frac{\operatorname{Hom}_{\mathbb{C}}\left(H^{3}(X, \mathbb{C})_{S_{b}}, H^{2}\left(S_{b}, \mathbb{C}\right) / j_{b}^{*} H^{2}(X, \mathbb{C})\right)}{F^{0} \operatorname{Hom}_{\mathbb{C}}(\cdot, \cdot) \oplus \operatorname{Hom}_{\mathbb{Z}}(\cdot, \cdot)},
$$

where $F^{\bullet}$ is the natural Hodge filtration on the homomorphism group, and $\mathrm{Hom}_{\mathbb{Z}}$ is the integral structure on the homomorphism group. Here

$$
\sigma_{F}: H^{3}(X, \mathbb{C})_{S_{b}} \rightarrow H^{3}\left(X, S_{b}, \mathbb{C}\right)
$$

and

$$
\sigma_{\mathbb{Z}}: H^{3}(X, \mathbb{Z})_{S_{b}} \rightarrow H^{3}\left(X, S_{b}, \mathbb{Z}\right)
$$


are splittings of the sequence

$$
0 \rightarrow \frac{H^{2}\left(S_{b}\right)}{j_{b}^{*} H^{2}(X)} \rightarrow H^{3}\left(X, S_{b}\right) \rightarrow H^{3}(X)_{S_{b}} \rightarrow 0
$$

which preserve the Hodge filtration and the integral structure, respectively.

The identification of the Jacobian (9) with its underlying real torus

$$
\frac{\operatorname{Hom}_{\mathbb{R}}\left(H^{3}(X, \mathbb{R})_{S_{b}}, H^{2}\left(S_{b}, \mathbb{R}\right) / j_{b}^{*} H^{2}(X, \mathbb{R})\right)}{\operatorname{Hom}_{\mathbb{Z}}\left(H^{3}(X, \mathbb{Z})_{S_{b}}, H^{2}\left(S_{b}, \mathbb{Z}\right) / j^{*} H^{2}(X, \mathbb{Z})\right)}
$$

comes from the identification

$$
\begin{aligned}
& \frac{\operatorname{Hom}_{\mathbb{C}}\left(H^{3}(X, \mathbb{C})_{S_{b}}, H^{2}\left(S_{b}, \mathbb{C}\right) / j_{b}^{*} H^{2}(X, \mathbb{C})\right)}{F^{0} \operatorname{Hom}_{\mathbb{C}}\left(H^{3}(X, \mathbb{C})_{S_{b}}, H^{2}\left(S_{b}, \mathbb{C}\right) / j_{b}^{*} H^{2}(X, \mathbb{C})\right)} \\
& \cong \operatorname{Hom}_{\mathbb{R}}\left(H^{3}(X, \mathbb{R})_{S_{b}}, H^{2}\left(S_{b}, \mathbb{R}\right) / j_{b}^{*} H^{2}(X, \mathbb{R})\right) .
\end{aligned}
$$

This isomorphism translates into the fact that there is one and only one real splitting that, when complexified, also preserves the Hodge filtration. We call this splitting $\sigma_{\mathbb{R}, F}$.

Hence we conclude that the Abel--Jacobi invariant we want to differentiate, which is the Abel-Jacobi invariant $\delta_{X, S_{b}}^{\prime}$ seen as an element of the real torus

$$
\operatorname{Hom}_{\mathbb{Z}}\left(H^{3}(X, \mathbb{Z})_{S_{b}}, H^{2}\left(S_{b}, \mathbb{Z}\right) / j^{*} H^{2}(X, \mathbb{Z})\right) \otimes_{\mathbb{Z}} \mathbb{R} / \mathbb{Z},
$$

is the class of

$$
\sigma_{\mathbb{R}, F}-\sigma_{\mathbb{Z}} \in \operatorname{Hom}_{\mathbb{R}}\left(H^{3}(X, \mathbb{R})_{S_{b}}, H^{2}\left(S_{b}, \mathbb{R}\right) / H^{2}(X, \mathbb{R})\right)
$$

in the quotient

$$
\frac{\operatorname{Hom}_{\mathbb{R}}\left(H^{3}(X, \mathbb{R})_{S_{b}}, H^{2}\left(S_{b}, \mathbb{R}\right) / j_{b}^{*} H^{2}(X, \mathbb{R})\right)}{\operatorname{Hom}_{\mathbb{Z}}\left(H^{3}(X, \mathbb{Z})_{S_{b}}, H^{2}\left(S_{b}, \mathbb{Z}\right) / j_{b}^{*} H^{2}(X, \mathbb{Z})\right)} .
$$

Now, starting from the cohomology class $[\omega] \in H^{3,0}(X)$, we have a class $\sigma_{\mathbb{R}, F}([\omega]) \in F^{3} H^{3}\left(X, S_{b}\right)$; on the other hand, since $j_{b}^{*} \omega=0$ on $S_{b}$ for all $b$, the $(3,0)$-form $\omega$ also determines a class in $H^{3}\left(X, S_{b}\right)$, which is easily seen to vary holomorphically with $b$, so that we get a section $\widetilde{\omega}$ of the bundle $\mathcal{H}_{X, \mathcal{S}}^{3,0}$, the holomorphic vector bundle over $B$ with fiber $H^{3,0}\left(X, S_{b}\right) \subset H^{3}\left(X, S_{b}, \mathbb{C}\right)$. We have

LEMMA 4.7. For any $b \in B$, the equality $\sigma_{\mathbb{R}, F, b}([\omega])=\widetilde{\omega}_{b}$ holds.

Proof of Lemma 4.7. We have $F^{3}\left(H^{2}\left(S_{b}\right) / j_{b}^{*} H^{2}(X)\right)=0$. Since the class $[\omega]$ belongs to $F^{3} H^{3}(X)$, any Hodge lifting of $[\omega]$ belongs to $F^{3} H^{3}\left(X, S_{b}\right)$. Since

$$
F^{3} H^{3}\left(X, S_{b}\right) \cap \frac{H^{2}\left(S_{b}\right)}{j_{b}^{*} H^{2}(X)}=F^{3} \frac{H^{2}\left(S_{b}\right)}{j_{b}^{*} H^{2}(X)}=0,
$$

the Hodge lifting of $[\omega]$ is unique. Hence $\widetilde{\omega}$ and $\sigma_{\mathbb{R}, F, b}$ must coincide. 
Recall (see Remark 4.1) that $d \delta_{\mathbb{C}}^{\prime}$ is the $\mathbb{C}$-linear extension of $\nabla \delta_{X, S_{b}}^{\prime}$, where $\nabla$ is the Gauss-Manin connection applied to the family of tori (11).

On the other hand, we have just shown that $\delta_{X, S_{b}}^{\prime}=\sigma_{F, \mathbb{R}}-\sigma_{\mathbb{Z}}$ modulo $\operatorname{Hom}_{\mathbb{Z}}\left(H^{3}(X, \mathbb{Z})_{S_{b}}, H^{2}\left(S_{b}, \mathbb{Z}\right) / j_{b}^{*} H^{2}(X, \mathbb{Z})\right)$. Since $\sigma_{\mathbb{Z}}$ is integral, hence flat, we get $\nabla \delta_{X, S_{b}}^{\prime}=\nabla \sigma_{F, \mathbb{R}}$.

Next, since $[\omega]$ gives a flat section of the bundle $\mathcal{H}_{\mathcal{S}}^{3}$ with fiber $H^{3}(X)_{S_{b}}$, we find that

$$
\nabla \sigma_{F, \mathbb{R}}([\omega])=\nabla^{X, S}\left(\sigma_{F, \mathbb{R}}([\omega])\right) .
$$

Here, on the right hand side, the Gauss-Manin connection acts on the bundle $\mathcal{H}_{X, S}^{3}$ of complex relative cohomology, with fiber $H^{3}\left(X, S_{b}\right)$ at $b \in B$, but the resulting form lies in

$$
\Omega_{B}^{\mathbb{C}} \otimes\left(\mathcal{H}_{\mathcal{S}}^{2} / \mathcal{H}_{X}^{2}\right) \subset \Omega_{B}^{\mathbb{C}} \otimes \mathcal{H}_{X, \mathcal{S}}^{3},
$$

because the projection of $\sigma_{F, \mathbb{R}}([\omega])$ in $\mathcal{H}_{\mathcal{S}}^{3}$ is flat. By Lemma 4.7 , we have $\sigma_{F, \mathbb{R}}([\omega])=\widetilde{\omega}$, and

$$
d \delta_{\mathbb{C}}^{\prime}([\omega])=\nabla \delta_{X, S_{b}}^{\prime}([\omega])=\nabla \sigma_{F, \mathbb{R}}([\omega])=\nabla^{X, \mathcal{S}}\left(\sigma_{F, \mathbb{R}}([\omega])\right)=\nabla^{X, \mathcal{S}}(\widetilde{\omega}) .
$$

The proof of the proposition will be complete once we have shown that

$$
\nabla^{X, \mathcal{S}}(\widetilde{\omega})=\alpha(\omega)
$$

This follows from a general fact concerning the Gauss-Manin connection acting on the relative cohomology of a family (cf. [Voi3]).

Let $\mathcal{X} \subset \mathcal{Y}$ be an immersion of two families of compact differentiable manifolds parametrized by a basis $B$. Let $\alpha$ be a closed differentiable $k$-form on $\mathcal{Y}$ which vanishes on each fiber $X_{b}$. Then $\alpha$ also provides a natural section $\widetilde{\alpha}$ of the bundle $\mathcal{H}_{\mathcal{Y}, \mathcal{X}}^{k}$ of relative cohomology. The projection of $\widetilde{\alpha}$ into $\mathcal{H}_{\mathcal{Y}}^{k}$ is flat, so that $\nabla \widetilde{\alpha}$ belongs to $\left(\mathcal{H}_{\mathcal{X}}^{k-1} / \mathcal{H}_{\mathcal{Y}}^{k-1}\right) \otimes \Omega_{B}^{\mathbb{C}}$. On the other hand, since $\alpha$ vanishes on $X_{b}$, the form $\alpha$ projects into a section of $A^{k-1}\left(X_{b}\right) \otimes \Omega_{B, b}$ for each $b \in B$, which is closed. Hence we get an element

$$
p\left(\alpha_{b}\right) \in H^{k-1}\left(X_{b}\right) \otimes \Omega_{B, b} .
$$

LEMMA 4.8. The projection of $p\left(\alpha_{b}\right)$ into $\left(H^{k-1}\left(X_{b}\right) / H^{k-1}\left(Y_{b}\right)\right) \otimes \Omega_{B, b}$ coincides with $\nabla \widetilde{\alpha}$ at any point $b \in B$.

This lemma shows equality (13) and completes the proof of Proposition 4.6.

STEP 2: The action of $d e_{\mathbb{C}}^{\prime}$. Recall that we have the map

$$
d e^{\prime} \wedge d f_{\mathbb{C}} \in \operatorname{Hom}_{\mathbb{C}}\left(H^{2}\left(S_{0}, \mathbb{C}\right)_{C_{0}}, \bigwedge^{2} \Omega_{B}^{\mathbb{C}}\right) .
$$

By (12) and (13) we have

$$
d \delta_{\mathbb{C}}^{\prime}([\omega])=\nabla^{X, \mathcal{S}}(\widetilde{\omega})=\alpha(\omega) \in \frac{\mathcal{H}_{\mathcal{S}}^{2,0}}{j^{*} \mathcal{H}_{X}^{2,0}} \otimes \Omega_{B}^{1},
$$


so that we can calculate $d e^{\prime} \wedge d f_{\mathbb{C}}\left(d \delta_{\mathbb{C}}^{\prime}([\omega])\right)$ as $d e^{\prime} \wedge d f_{\mathbb{C}}(\alpha(\omega))$. Now, since $\alpha(\omega)$ is the projection of $p\left(j^{*} \omega\right) \in \mathcal{H}_{\mathcal{S}}^{2,0} \otimes \Omega_{B}$ in the quotient $\left(\mathcal{H}_{\mathcal{S}}^{2,0} / \mathcal{H}_{X}^{2,0}\right) \otimes \Omega_{B}$, we have as well

$$
d e^{\prime} \wedge d f_{\mathbb{C}}\left(d \delta_{\mathbb{C}}^{\prime}([\omega])\right)=d e^{\prime} \wedge d f_{\mathbb{C}}\left(p\left(j^{*} \omega\right)\right)
$$

We begin the computation again by writing

$$
d e^{\prime} \in \frac{H^{1}\left(C_{0}, \mathbb{Z}\right)}{i_{0}^{*} H^{1}\left(S_{0}, \mathbb{Z}\right)} \otimes_{\mathbb{Z}} \frac{H^{2}\left(S_{0}, \mathbb{Z}\right)}{\left\langle C_{0, r}\right\rangle} \otimes_{\mathbb{Z}} \Omega_{B}^{\mathbb{R}}
$$

as a homomorphism (after $\mathbb{C}$-linear extension)

$$
d e_{\mathbb{C}}^{\prime} \in \operatorname{Hom}_{\mathbb{C}}\left(H^{2}\left(S_{0}, \mathbb{C}\right)_{C_{0}}, \frac{H^{1}\left(C_{0}, \mathbb{C}\right)}{i_{0}^{*} H^{1}\left(S_{0}, \mathbb{C}\right)} \otimes_{\mathbb{C}} \Omega_{B}^{\mathbb{C}}\right) .
$$

This map in turn defines an extended homomorphism

$$
d e_{\mathbb{C}, 1}^{\prime} \in \operatorname{Hom}_{\mathbb{C}}\left(H^{2}\left(S_{0}, \mathbb{C}\right)_{C_{0}} \otimes_{\mathbb{C}} \Omega_{B}^{\mathbb{C}}, \frac{H^{1}\left(C_{0}, \mathbb{C}\right)}{i_{0}^{*} H^{1}\left(S_{0}, \mathbb{C}\right)} \otimes_{\mathbb{C}} \wedge^{2} \Omega_{B}^{\mathbb{C}}\right)
$$

by the rule

$$
d e_{\mathbb{C}, 1}^{\prime}\left(\alpha \otimes_{\mathbb{C}} \eta\right)=d e_{\mathbb{C}}^{\prime}(\alpha) \wedge \eta
$$

Similarly, we have

$$
d f \in\left(\operatorname{ker} i_{0 *}: H^{1}\left(C_{0}, \mathbb{C}\right) \rightarrow H^{3}\left(S_{0}, \mathbb{C}\right)\right) \otimes_{\mathbb{C}} \Omega_{B}^{\mathbb{C}},
$$

which we view as a homomorphism

$$
d f_{\mathbb{C}} \in \operatorname{Hom}\left(\frac{H^{1}\left(C_{0}, \mathbb{C}\right)}{i_{0}^{*} H^{1}\left(S_{0}, \mathbb{C}\right)}, \Omega_{B}^{\mathbb{C}}\right),
$$

which can be extended to

$$
d f_{\mathbb{C}, 2} \in \operatorname{Hom}\left(\frac{H^{1}\left(C_{0}, \mathbb{C}\right)}{i_{0}^{*} H^{1}\left(S_{0}, \mathbb{C}\right)} \otimes_{\mathbb{C}} \bigwedge^{2} \Omega_{B}^{\mathbb{C}}, \bigwedge^{3} \Omega_{B}^{\mathbb{C}}\right)
$$

by the rule

$$
d f_{\mathbb{C}, 2}\left(\alpha \otimes_{\mathbb{C}} \eta\right)=d f_{\mathbb{C}}(\alpha) \wedge \eta .
$$

It is clear from these definitions that

$$
d e^{\prime} \wedge d f_{\mathbb{C}}\left(p\left(j^{*} \omega\right)\right)=-d f_{\mathbb{C}, 2}\left(d e_{\mathbb{C}, 1}^{\prime}\left(p\left(j^{*} \omega\right)\right)\right) .
$$

(Notice that we are not allowed to write this equality with $p\left(j^{*} \omega\right)$ replaced by $\alpha(\omega)$, since $d e_{\mathbb{C}, 1}^{\prime}$ does not act on $\alpha(\omega)$.)

So first we have to compute $d e_{\mathbb{C}, 1}^{\prime}\left(p\left(j^{*} \omega\right)\right)$. On $\mathcal{C}$ we have a natural surjective morphism

$$
q: \Omega_{\mathcal{C}}^{3} \rightarrow \Omega_{\mathcal{C} / B} \otimes \pi^{*} \Omega_{B}^{2}
$$

By pulling back via $j \circ i$, the holomorphic 3 -form $\omega$ on $X$ gives a section $(j \circ i)^{*} \omega$ of $\Omega_{\mathcal{C}}^{3}$; we project it via $q$ to the section $q\left((j \circ i)^{*} \omega\right)$ of $\Omega_{\mathcal{C} / B}^{1} \otimes \pi^{*} \Omega_{B}^{2}$, 
further via $\pi_{*}$ to a section still denoted by $q\left((j \circ i)^{*} \omega\right)$ of

$$
\pi_{*} \Omega_{\mathcal{C} / B}^{1} \otimes \pi_{*} \pi^{*} \Omega_{B}^{2}=\mathcal{H}_{\mathcal{C}}^{1,0} \otimes \Omega_{B}^{2} \subseteq \mathcal{H}_{\mathcal{C}}^{1} \otimes \Omega_{B}^{2},
$$

and then to a section

$$
\beta(\omega) \in \frac{\mathcal{H}_{\mathcal{C}}^{1,0}}{i^{*} \mathcal{H}_{\mathcal{S}}^{1,0}} \otimes \Omega_{B}^{2}
$$

Here $\mathcal{H}_{\mathcal{C}}^{1,0}$ and $\mathcal{H}_{\mathcal{S}}^{1,0}$ denote the Hodge bundles with fibers $H^{1,0}\left(C_{b}\right)$ and $H^{1,0}\left(S_{b}\right)$, respectively.

Proposition 4.9. Let $\omega$ be a holomorphic 3 -form on $X$. Then for any $b \in B$ we have the equality

$$
d e_{\mathbb{C}, 1}^{\prime}\left(p\left(j^{*} \omega\right)\right)_{b}=\beta(\omega)_{b}
$$

via the inclusion

$$
\frac{H^{1,0}\left(C_{b}, \mathbb{C}\right)}{i_{b}^{*} H^{1,0}\left(S_{b}, \mathbb{C}\right)} \otimes_{\mathbb{C}} \Omega_{B, b}^{2} \subseteq \frac{H^{1}\left(C_{b}, \mathbb{C}\right)}{i_{b}^{*} H^{1}\left(S_{b}, \mathbb{C}\right)} \otimes_{\mathbb{C}} \Omega_{B, b}^{2, \mathbb{C}} \cong \frac{H^{1}\left(C_{0}, \mathbb{C}\right)}{i_{0}^{*} H^{1}\left(S_{b}, \mathbb{C}\right)} \otimes_{\mathbb{C}} \Omega_{B, b}^{2, \mathbb{C}}
$$

Proof. Carlson's description of $e_{S_{b}, C_{b}}^{\prime}$ as an extension class says it is the class of $\sigma_{F}^{\prime}-\sigma_{\mathbb{Z}}^{\prime}$ in the quotient

$$
\frac{\operatorname{Hom}_{\mathbb{C}}\left(H^{2}\left(S_{b}, \mathbb{C}\right)_{C_{b}}, H^{1}\left(C_{b}, \mathbb{C}\right) / i_{b}^{*} H^{1}\left(S_{b}, \mathbb{C}\right)\right)}{F^{0} \operatorname{Hom}_{\mathbb{C}}(\cdot, \cdot)+\operatorname{Hom}_{\mathbb{Z}}(\cdot, \cdot)},
$$

where $\sigma_{F}^{\prime}$ and $\sigma_{\mathbb{Z}}^{\prime}$ are Hodge and integral splittings of the sequence

$$
0 \rightarrow H^{1}\left(C_{b}\right) / j_{b}^{*} H^{1}\left(S_{b}\right) \rightarrow H^{2}\left(S_{b}, C_{b}\right) \rightarrow H^{2}\left(S_{b}\right)_{C_{b}} \rightarrow 0
$$

of mixed Hodge structures.

Just as in the previous step we find that $e_{S_{b}, C_{b}}^{\prime}$ is the class of $\sigma_{\mathbb{R}, F}^{\prime}-\sigma_{\mathbb{Z}}^{\prime}$ in the quotient

$$
\begin{aligned}
& \frac{\operatorname{Hom}_{\mathbb{Z}}\left(H^{2}\left(S_{b}, \mathbb{Z}\right)_{C_{b}}, H^{1}\left(C_{b}, \mathbb{R}\right) / i_{b}^{*} H^{1}\left(S_{b}, \mathbb{R}\right)\right)}{\operatorname{Hom}_{\mathbb{Z}}\left(H^{2}\left(S_{b}, \mathbb{Z}\right)_{C_{b}}, H^{1}\left(C_{b}, \mathbb{Z}\right) / i_{b}^{*} H^{1}\left(S_{b}, \mathbb{Z}\right)\right)} \\
& \cong \operatorname{Hom}_{\mathbb{Z}}\left(H^{2}\left(S_{b}, \mathbb{Z}\right)_{C_{b}}, H^{1}\left(C_{b}, \mathbb{Z}\right) / i_{b}^{*} H^{1}\left(S_{b}, \mathbb{Z}\right)\right) \otimes_{\mathbb{Z}} \mathbb{R} / \mathbb{Z},
\end{aligned}
$$

where $\sigma_{\mathbb{R}, F}^{\prime}$ is the unique real splitting of (17) that also preserves the Hodge filtration.

Now, by the above we have

$$
d e^{\prime}=\nabla\left(\sigma_{F, \mathbb{R}}^{\prime}-\sigma_{\mathbb{Z}}^{\prime}\right)=\nabla \sigma_{F, \mathbb{R}}^{\prime},
$$

since $\sigma_{\mathbb{Z}}^{\prime}$ is an integral, hence flat section of the bundle with fiber

$$
\operatorname{Hom}\left(H^{2}\left(S_{b}\right)_{C_{b}}, H^{1}\left(C_{b}\right) / i_{b}^{*} H^{1}\left(S_{b}\right)\right) \text {. }
$$

In what follows we shall denote by $\mathcal{H}_{\mathcal{C}}^{2} \subset \mathcal{H}_{\mathcal{S}}^{2}$ the flat subbundle with fiber $H^{2}\left(S_{b}\right)_{C_{b}}$ and by $\mathcal{H}_{\mathcal{S}, \mathcal{C}}^{2}$ the flat bundle with fiber $H^{2}\left(S_{b}, C_{b}\right)$. The GaussManin connection acts in a compatible way on all of these bundles. 
Since $p(\omega)$ is a section of $\mathcal{H}_{\mathcal{C}}^{2} \otimes \Omega_{B}$ which is closed with respect to the Gauss-Manin connection, it follows from Leibniz's rule (15) and the formula (18) that

$$
d e_{\mathbb{C}, 1}^{\prime}\left(p\left(j^{*} \omega\right)\right)=\left(\nabla \sigma_{F, \mathbb{R}}^{\prime}\right)\left(p\left(j^{*} \omega\right)\right)=\nabla\left(\sigma_{F, \mathbb{R}}^{\prime}(p(\omega))\right),
$$

where $\nabla$ on the right hand side acts on the bundle $\mathcal{H}_{\mathcal{S}, \mathcal{C}}^{2}$ of relative cohomology.

Now, the argument in the proof of Lemma 4.7 gives as well

Lemma 4.10. If $\alpha \in H^{2,0}\left(S_{b}\right)$, the class $\sigma_{F, \mathbb{R}}^{\prime}(\alpha) \in H^{2}\left(S_{b}, C_{b}, \mathbb{C}\right)$ is the class of the closed form $\alpha$, which vanishes on $C_{b}$.

It follows from this lemma that $\sigma_{F, \mathbb{R}}^{\prime}\left(p\left(j^{*} \omega\right)\right) \in \mathcal{H}_{\mathcal{S}, \mathcal{C}}^{2} \otimes \Omega_{B}^{\mathbb{C}}$ is equal to $\widetilde{p(\omega)}$, where the tilde denotes the lifting $H^{2,0}\left(S_{b}\right) \rightarrow H^{2}\left(S_{b}, C_{b}\right)$ described in the statement of the lemma. Combining (19) and the lemma above, we get

$$
d e_{\mathbb{C}, 1}^{\prime}\left(p\left(j^{*} \omega\right)\right)=\nabla\left(\widetilde{p\left(j^{*} \omega\right)}\right),
$$

where $\nabla$ on the right hand side acts on $\mathcal{H}_{\mathcal{S}, \mathcal{C}}^{2} \otimes \Omega_{B}^{\mathbb{C}}$, but the resulting differential lies in $\left(\mathcal{H}_{\mathcal{C}}^{1} / i^{*} \mathcal{H}_{\mathcal{S}}^{1}\right) \otimes \bigwedge^{2} \Omega_{B}^{\mathbb{C}}$, because the projection of $\widetilde{p\left(j^{*} \omega\right)}$ into $\mathcal{H}_{\mathcal{C}}^{2} \otimes \Omega_{B}^{\mathbb{C}}$ is $\nabla$-closed.

The proof of Proposition 4.9 is then concluded by the following

Lemma 4.11. We have the equality

$$
\nabla\left(\widetilde{p\left(j^{*} \omega\right)}\right)=\beta(\omega)
$$

in $\left(\mathcal{H}_{\mathcal{C}}^{1} / i^{*} \mathcal{H}_{\mathcal{S}}^{1}\right) \otimes \bigwedge^{2} \Omega_{B}^{\mathbb{C}}$.

This lemma follows from a slight generalization of Lemma 4.8, which concerned the Gauss-Manin connection acting on the relative cohomology of a family of pairs. Remember that $p\left(j^{*} \omega\right) \in \mathcal{H}_{\mathcal{S}}^{2} \otimes \Omega_{B}$ is obtained from the 3 -form $j^{*} \omega$ on $\mathcal{S}$ by observing that $j^{*} \omega$ is closed and in $L^{1} A_{\mathcal{S}}^{3}$-here $L^{\bullet} A_{\mathcal{S}}^{\bullet}$ denotes the Leray filtration on the sheaf of differential forms on $\mathcal{S}$ (cf. [Voi2]). Now the form $i^{*}\left(j^{*} \omega\right)$ on $\mathcal{C}$ belongs to $L^{2} A_{\mathcal{C}}^{3}$, so this provides on the one hand the lifting $\widetilde{p\left(j^{*} \omega\right)}$ of $p\left(j^{*} \omega\right)$ to $\mathcal{H}_{S, C}^{2} \otimes \Omega_{B}$, and on the other hand the projection $q\left(i^{*}\left(j^{*} \omega\right)\right) \in \mathcal{H}_{\mathcal{C}}^{1} \otimes \Omega_{B}^{2}$. The equality (21) is then essentially the same statement as the equality given in Lemma 4.8, just for the $L^{2}$ level of the Leray filtration. This completes the proof of Proposition 4.9.

STEP 3: The action of $d f_{\mathbb{C}}$. In this third and last step we calculate $d e^{\prime} \wedge d f_{\mathbb{C}}\left(d \delta_{\mathbb{C}}^{\prime}([\omega])\right)$, that is, Green's pull-back of $\omega$. We already noted that

$$
d e^{\prime} \wedge d f_{\mathbb{C}}\left(d \delta_{\mathbb{C}}^{\prime}([\omega])\right)=-d f_{\mathbb{C}, 2}\left(d e_{\mathbb{C}, 1}^{\prime}\left(p\left(j^{*} \omega\right)\right) .\right.
$$


Since by Proposition 4.9 we know that

$$
d e_{\mathbb{C}, 1}^{\prime}\left(p\left(j^{*} \omega\right)\right)=\beta(\omega) \in \frac{\mathcal{H}_{\mathcal{C}}^{1,0}}{i^{*} \mathcal{H}_{\mathcal{S}}^{1,0}} \otimes \Omega_{B}^{2},
$$

we do this by computing $d f_{\mathbb{C}, 2}(\beta(\omega))$. Recall that $\beta(\omega)$ is the projection of $q\left(i^{*}\left(j^{*} \omega\right)\right) \in \Omega_{\mathcal{C} / B} \otimes \bigwedge^{2} \Omega_{B}$ in $\left(\mathcal{H}_{\mathcal{C}}^{1,0} / i^{*} \mathcal{H}_{\mathcal{S}}^{1,0}\right) \otimes \Omega_{B}^{2}$. It follows that we have in fact $d f_{\mathbb{C}, 2}(\beta(\omega))=d f_{\mathbb{C}, 2}(q(\omega))$. Theorem 4.5 then follows from this last result:

Proposition 4.12. Let $\omega^{\prime}$ be a form of type $(3,0)$ on $\mathcal{C}$, and $q\left(\omega^{\prime}\right)$ the section of $\mathcal{H}_{\mathcal{C}}^{1,0} \otimes \Omega_{B}^{2}$ associated to it. Then

$$
d f_{\mathbb{C}, 2}(q(\omega))=\sum_{i=0}^{N} m_{i} s_{i}^{*}\left(\omega^{\prime}\right) \in \Omega_{B}^{3} .
$$

This proposition is proved exactly as in [Voi3, Lemma 8], where it is stated for a 2 -form on $\mathcal{C}$.

5. On the image of the map $\psi_{3}^{3}$. In this section we use Theorem 4.5 comparing Mumford's and Green's pull-backs to prove the following result:

Theorem 5.1. Let $X$ be a 3-fold with $h^{1,0}(X)=b_{4, \operatorname{tr}}(X)=0$ (this implies that the maps $\operatorname{alb}_{X}$ and $\psi_{2}^{3}$ are identically zero). Then if

$$
h^{3,0}(X):=\operatorname{dim} H^{0}\left(\Omega_{X}^{3}\right)>0,
$$

the map $\psi_{3}^{3}: \operatorname{ker}\left(\psi_{2}^{3}\right) \rightarrow J_{3}^{3}(X)$ has, modulo torsion, positive-dimensional image.

Note that the conditions are fulfilled, for example, by 3-dimensional complete intersections of hypersurfaces in projective space in view of Lefschetz's hyperplane theorem.

The theorem is obtained by arguing that if $\psi_{3}^{3}$ is 0 , then we can find a diagram as at the beginning of the previous section, with

$$
(j \circ i)_{*} Z_{b}=x_{b}-y_{b},
$$

$\delta_{b}^{\prime} \cdot e_{b} \cdot f_{b}=0$ for any $b$, and the map $b \mapsto\left(x_{b}, y_{b}\right) \in X \times X$ is submersive. One can then apply Theorem 4.5 together with Proposition 5.4 below to conclude that $H^{3,0}(X)=0$.

More generally, Mumford's technique of pulling back a holomorphic form on the symmetric product $X^{(k)} \times X^{(k)}$ of the variety $X$, induced by a holomorphic form on $X$, to a variety parametrizing 0 -cycles, in order to bound the dimension of the fibers of a map defined on this symmetric product, can be combined with the reasoning above to prove that if $H^{3,0}(X) \neq 0$, then the image of $\psi_{3}^{3}$ is actually infinite-dimensional. 
We work with the diagram from the beginning of the last section, in which the 0-cycles, curves and surfaces are parametrized by a complex ball $B$. The first step in the proof of the theorem is the following result:

Proposition 5.2. Let $V \subset B$ be a smooth, real-analytic subset such that for all $b \in V$, the Green contraction

$$
\delta_{b}^{\prime} \cdot e_{b} \cdot f_{b} \in J^{3}(X)_{\mathrm{AJ}} \otimes_{\mathbb{Z}}(\mathbb{R} / \mathbb{Z})^{\otimes 2}
$$

is torsion. Then for any holomorphic 3 -form $\omega$ on $X$, the 3 -form $d \delta^{\prime} \wedge d e^{\prime} \wedge$ $d f([\omega])$ defined in the previous section vanishes on $V$.

Here $\delta_{b}^{\prime}=\delta_{X, S_{b}}^{\prime}, e_{b}=e_{S_{b}, C_{b}}$, and $f_{b}=f_{C_{b}, Z_{b}}$.

Proof. To make the calculation a bit simpler, we will assume that the surface $S=S_{0}$ is regular, i.e. that $H^{1}(S, \mathbb{Z})=0$. Let $C=C_{0}$ and let

$$
\left\{\alpha_{i}, \beta_{i}\right\}, \quad i=1, \ldots, g,
$$

be a symplectic basis for $H^{1}(C, \mathbb{Z})$. Let

$$
\left\{\gamma_{j}\right\}, j=1, \ldots, b_{2}(S), \quad \text { and } \quad\left\{\gamma_{j}^{*}\right\}, j=1, \ldots, b_{2}(S)_{\text {new }},
$$

be respectively a basis of $H^{2}(S, \mathbb{Z})$ such that $\left\{\gamma_{j}\right\}, j=1, \ldots, b_{2}(S)_{\text {new }}$, induces a basis of

$$
H^{2}(S, \mathbb{Z})_{\text {new }}=\operatorname{ker}\left(j_{*}: H^{2}(S, \mathbb{Z}) \rightarrow H^{4}(X, \mathbb{Z})\right),
$$

and the dual basis of $H^{2}(S, \mathbb{Z})_{\text {new }}^{*}=H^{2}(S, \mathbb{Z}) / j^{*} H^{2}(X, \mathbb{Z})$. Hence by definition we have $\left\langle\gamma_{i}, \gamma_{j}^{*}\right\rangle=0$ for $i \neq j, i \leq b_{2}(S)_{\text {new }}$ and $\left\langle\gamma_{j}, \gamma_{j}^{*}\right\rangle=1$, where $\langle\cdot, \cdot\rangle$ is the duality between $H^{2}(S, \mathbb{Z})_{\text {new }}$ and $H^{2}(S, \mathbb{Z}) / j^{*} H^{2}(X, \mathbb{Z})$. Finally, let

$$
\left\{\delta_{n}\right\}, \quad n=1, \ldots, b_{3}(X),
$$

be a basis of

$$
H^{3}(X, \mathbb{Z})_{S}^{*}=\frac{H^{3}(X, \mathbb{Z})}{j_{*} H^{1}(S, \mathbb{Z})},
$$

which is equal to $H^{3}(X, \mathbb{Z})$ by our assumption that $S$ is regular. Then we can write explicitly

$$
\begin{aligned}
& f_{b}=\sum_{i=1}^{g} \phi_{i}(b) \otimes_{\mathbb{Z}} \alpha_{i}+\sum_{i=1}^{g} \psi_{i}(b) \otimes_{\mathbb{Z}} \beta_{i} \in \mathbb{R} / \mathbb{Z} \otimes_{\mathbb{Z}} H^{1}(C, \mathbb{Z}), \\
& e_{b}^{\prime}=\sum_{j=1}^{b_{2}(S)}\left\{\sum_{i=1}^{g} \varrho_{i j}(b) \otimes_{\mathbb{Z}} \gamma_{j} \otimes_{\mathbb{Z}} \alpha_{i}+\sum_{i=1}^{g} \chi_{i j}(b) \otimes_{\mathbb{Z}} \gamma_{j} \otimes_{\mathbb{Z}} \beta_{i}\right\},
\end{aligned}
$$

modulo

$$
\mathbb{R} / \mathbb{Z} \otimes_{\mathbb{Z}} \mathrm{NS}(S) \otimes_{\mathbb{Z}} H^{1}(C, \mathbb{Z})
$$


and

$$
\delta_{b}^{\prime}=\sum_{n=1}^{b_{3}(X)} \sum_{j=1}^{b_{2}(S)_{\mathrm{new}}} \eta_{n j}(b) \otimes_{\mathbb{Z}} \delta_{n} \otimes_{\mathbb{Z}} \gamma_{j}^{*},
$$

where $\phi_{i}, \psi_{i}, \varrho_{i j}, \chi_{i j}$ and $\eta_{n j}: B \rightarrow \mathbb{R} / \mathbb{Z}$ are differentiable functions.

By assumption, Green's contraction $e_{b}^{\prime} \cdot f_{b}$ belongs to

$$
\operatorname{ker}\left(j_{*}: \frac{H^{2}(S, \mathbb{Z})}{\mathrm{NS}(S)} \rightarrow \frac{H^{4}(X, \mathbb{Z})}{j_{*} \mathrm{NS}(S)}\right)
$$

which is isomorphic to $H^{2}(S, \mathbb{Z})_{\text {new }} /\left(\operatorname{NS}(S) \cap\right.$ ker $\left.j_{*}\right)$. This means that

$$
\sum_{j=1}^{b_{2}(S)}\left(\sum_{i=1}^{g} \varrho_{i j}(b) \otimes_{\mathbb{Z}} \psi_{i}(b)-\sum_{i=1}^{g} \chi_{i j}(b) \otimes_{\mathbb{Z}} \phi_{i}(b)\right) \otimes_{\mathbb{Z}} \gamma_{j}
$$

belongs to $(\mathbb{R} / \mathbb{Z})^{\otimes 2} \otimes_{\mathbb{Z}} H^{2}(S, \mathbb{Z})_{\text {new }}$ modulo $(\mathbb{R} / \mathbb{Z})^{\otimes 2} \otimes_{\mathbb{Z}} \mathrm{NS}(S)$. Let us write

$$
\sum_{j=1}^{b_{2}(S)}\left(\sum_{i=1}^{g} \varrho_{i j}(b) \otimes_{\mathbb{Z}} \psi_{i}(b)-\sum_{i=1}^{g} \chi_{i j}(b) \otimes_{\mathbb{Z}} \phi_{i}(b)\right) \otimes_{\mathbb{Z}} \gamma_{j}=a+c
$$

with $a \in(\mathbb{R} / \mathbb{Z})^{\otimes 2} \otimes_{\mathbb{Z}} H^{2}(S, \mathbb{Z})_{\text {new }}$, and $c \in(\mathbb{R} / \mathbb{Z})^{\otimes 2} \otimes_{\mathbb{Z}} \mathrm{NS}(S)$. Then

$$
a=\sum_{j=1}^{b_{2}(S)_{\text {new }}}\left(\sum_{i=1}^{g} \varrho_{i j}(b) \otimes_{\mathbb{Z}} \psi_{i}(b)-\sum_{i=1}^{g} \chi_{i j}(b) \otimes_{\mathbb{Z}} \phi_{i}(b)\right) \otimes_{\mathbb{Z}} \gamma_{j}
$$

modulo $(\mathbb{R} / \mathbb{Z})^{\otimes 2} \otimes_{\mathbb{Z}}\left(\mathrm{NS}(S) \cap \operatorname{ker} j_{*}\right)$. Furthermore, by the definition of our contraction $\delta_{b}^{\prime} \cdot e_{b} \cdot f_{b}$, it is equal (modulo $(\mathbb{R} / \mathbb{Z})^{\otimes 2} \otimes_{\mathbb{Z}} \operatorname{im}\left(\mathrm{AJ}_{X}\right)$ ) to the contraction $\delta_{b}^{\prime} \cdot a$ obtained by making use of the pairing between $H^{2}(S, \mathbb{Z})_{\text {new }}$ and $H^{2}(S, \mathbb{Z}) / j^{*} H^{2}(X, \mathbb{Z})$. Hence

$$
\begin{aligned}
\delta_{b}^{\prime} \cdot e_{b} \cdot f_{b}= & \sum_{n=1}^{b_{3}(X)} \sum_{j=1}^{b_{2}(S)_{\text {new }}} \eta_{n j}(b) \\
& \otimes_{\mathbb{Z}}\left\{\sum_{i=1}^{g} \varrho_{i j}(b) \otimes_{\mathbb{Z}} \psi_{i}(b)-\sum_{i=1}^{g} \chi_{i j}(b) \otimes_{\mathbb{Z}} \phi_{i}(b)\right\} \otimes_{\mathbb{Z}} \delta_{n}
\end{aligned}
$$

modulo $(\mathbb{R} / \mathbb{Z})^{\otimes 2} \otimes_{\mathbb{Z}} \operatorname{im}\left(\mathrm{AJ}_{X}\right)$.

We have to show that if the expression (22) vanishes modulo torsion in $J^{3}(X)_{\mathrm{AJ}} \otimes_{\mathbb{Z}}(\mathbb{R} / \mathbb{Z})^{\otimes 2}$ for any point $b$ of $V$, then for any $\omega \in H^{3,0}(X)$, the 3 -form $d \delta \wedge d e \wedge d f([\omega])$ defined on $B$ vanishes on $V$.

First of all, we note that this assumption means that for any $v \in V$ there exists a non-zero integer $m \in \mathbb{N}$ such that $m \delta_{b}^{\prime} \cdot e_{b}^{\prime} \cdot f_{b}=0$. The formula for $\delta_{b}^{\prime} \cdot e_{b}^{\prime} \cdot f_{b}$ now shows that the locus $V_{m} \subset V$ where $m \delta_{b}^{\prime} \cdot e_{b}^{\prime} \cdot f_{b}=0$ is a countable union of real-analytic subsets of $V$. The assumption says that $V$ 
is the union over $m$ of the $V_{m}$ 's and by Baire's theorem it follows that $V$ must be equal to some $V_{m}$. So we may assume that $m \delta_{b}^{\prime} \cdot e_{b}^{\prime} \cdot f_{b}=0$ on $V$.

The image of the Abel-Jacobi map. The image $\mathrm{im}\left(\mathrm{AJ}_{X}\right)$ of the AbelJacobi map is a subgroup of $J^{3}(X)$; it is an extension of an Abelian subvariety $J^{3}(X)$ alg of $J^{3}(X)$, its connected component of 0 , by a countable group $\operatorname{Griff}(X) . J^{3}(X)$ alg corresponds to a certain real subtorus

$$
H^{3}(X, \mathbb{Z})_{\text {alg }} \otimes_{\mathbb{Z}} \mathbb{R} / \mathbb{Z} \subset H^{3}(X, \mathbb{Z}) \otimes_{\mathbb{Z}} \mathbb{R} / \mathbb{Z}=J^{3}(X),
$$

where the sublattice $H^{3}(X, \mathbb{Z})$ alg $\subset H^{3}(X, \mathbb{Z})$ has the property that the complexification $H^{3}(X, \mathbb{Z})_{\text {alg }} \otimes_{\mathbb{Z}} \mathbb{C} \subset H^{3}(X, \mathbb{C})$ is contained in $H^{2,1}(X) \oplus$ $H^{1,2}(X)$, and thus is perpendicular to $H^{3,0}(X)$ with respect to Poincaré duality.

A special case. Assume first that the stronger condition

$$
m \delta_{b}^{\prime} \cdot e_{b}^{\prime} \cdot f_{b}=0 \quad \text { in }\left(J^{3}(X) / J^{3}(X)_{\text {alg }}\right) \otimes_{\mathbb{Z}}(\mathbb{R} / \mathbb{Z})^{\otimes 2}
$$

is satisfied along $V$. We may suppose that the basis $\delta_{n}$ has been chosen so that the

$$
\delta_{k}, \quad k \leq b_{3}(X)_{\text {alg }}:=\operatorname{rk} H^{3}(X, \mathbb{Z})_{\text {alg }},
$$

induce a basis of $H^{3}(X, \mathbb{Z})_{\mathrm{alg}}$. Then from $(22)$ we conclude that

$$
\begin{aligned}
m \sum_{\left.n=b_{3}(X)\right)_{\mathrm{alg}}+1}^{b_{3}(X)} & \sum_{j=1}^{b_{2}(S)_{\text {new }}} \eta_{n j}(b) \\
& \otimes_{\mathbb{Z}}\left\{\sum_{i=1}^{g} \varrho_{i j}(b) \otimes_{\mathbb{Z}} \psi_{i}(b)-\sum_{i=1}^{g} \chi_{i j}(b) \otimes_{\mathbb{Z}} \phi_{i}(b)\right\} \otimes_{\mathbb{Z}} \delta_{n}
\end{aligned}
$$

vanishes in $\left(H^{3}(X, \mathbb{Z}) / H^{3}(X, \mathbb{Z})_{\text {alg }}\right) \otimes_{\mathbb{Z}}(\mathbb{R} / \mathbb{Z})^{\otimes 3}$. Now we observe that since $[\omega]$ is perpendicular to $\delta_{n}, n \leq b_{3}(X)_{\text {alg }}$, we have

$$
d \delta_{\mathbb{C}}^{\prime}([\omega])=\sum_{n=b_{3}(X)_{\mathrm{alg}}+1}^{b_{3}(X)} \sum_{j=1}^{b_{2}(S)_{\text {new }}} d \eta_{n j}(b)\left\langle\delta_{n},[\omega]\right\rangle \otimes \gamma_{j}^{*}
$$

in $H^{2}(S, \mathbb{C})_{\text {new }} \otimes_{\mathbb{C}} \Omega_{B}^{\mathbb{C}}$. It follows that the coefficients $\eta_{n j}$ with $n \leq b_{3}(X)_{\text {alg }}$ will not play any role in the computation of $d \delta \wedge d e \wedge d f([\omega])$.

Suppose we start out with finitely many real vector spaces $V_{1}, \ldots, V_{k}$, all of finite dimension, and that we have a relation $F=0$ in $V_{1} \otimes_{\mathbb{Q}} \cdots \otimes_{\mathbb{Q}}$ $V_{k} \otimes_{\mathbb{Q}}(\mathbb{R} / \mathbb{Q})^{\otimes k}$. Taking $\mathbb{R}$-bases for the vector spaces this gives a number of equations in $(\mathbb{R} / \mathbb{Q})^{\otimes k}$ together equivalent to the original relation $F=0$.

Suppose next that the vector spaces are the stalks at $v \in V$ of a local system of $\mathbb{R}$-vector spaces. Gauss-Manin allows one to differentiate smooth sections in the corresponding vector bundle. For computations, $B$ is a small ball so that the local systems can be considered to be trivialized in a flat 
frame and the Gauss-Manin connection then amounts to differentiating the coefficients in this frame. We also suppose that the relation extends over $V \subset B$, a real-analytic submanifold, so that it can be differentiated. Say we have

$$
\sum_{I} v_{i_{1}}(v) \otimes_{\mathbb{Q}} \cdots \otimes_{\mathbb{Q}} v_{i_{k}}(v)=0
$$

where $I=\left(i_{1}, \ldots, i_{k}\right)$, and the $v_{i_{j}}(v)$ are smooth sections of $V_{j} \otimes_{\mathbb{Q}}(\mathbb{R} / \mathbb{Q})$ (see Proposition 3 and its proof in [Voi3]). As we saw above, the relation is equivalent to equations of the form

$$
\sum_{I} r_{i_{1}} \otimes_{\mathbb{Q}} \cdots \otimes_{\mathbb{Q}} r_{i_{k}}=0
$$

where $I=\left(i_{1}, \ldots, i_{k}\right)$, and the $r_{i_{j}}$ are smooth functions on $V$ with values in $\mathbb{R} / \mathbb{Q}$. One wants to see that there is a relation

$$
\sum_{I} d v_{i_{1}}(v) \wedge \cdots \wedge d v_{i_{k}}(v)=0
$$

inside $\Omega_{V}^{k}$. This follows inductively by using the next result:

LEMMA 5.3. Let $\omega_{\lambda}$ be a smooth section of $\Omega_{V}^{a} \otimes_{\mathbb{Q}}(\mathbb{R} / \mathbb{Q})^{\otimes b}$, and $e_{\lambda} a$ smooth $\mathbb{R} / \mathbb{Q}$-valued function on $V$ such that

$$
\sum_{\lambda \in I} \omega_{\lambda} \otimes_{\mathbb{Q}} e_{\lambda}=0
$$

in each stalk of $\Omega_{V}^{a} \otimes_{\mathbb{Q}}(\mathbb{R} / \mathbb{Q})^{\otimes(b+1)}$. Then

$$
\sum_{\lambda \in I} \omega_{\lambda} \otimes_{\mathbb{Q}} d e_{\lambda}=0 \quad \text { in } \Omega_{V}^{a+1} \otimes_{\mathbb{Q}}(\mathbb{R} / \mathbb{Q})^{\otimes b} .
$$

Proof. First we may assume that $V$ is connected. Then, by a Baire category argument, there is a dense subset $V^{0}$ of $V$ with the property that any relation $\sum a_{i} e_{i}(v)=0$ over $V^{0}$ holds over $V$. Now pick a subset $J \subset I$ such that $e_{j}\left(v_{0}\right)$ gives a $\mathbb{Q}$-basis for the $\mathbb{Q}$-span of the $e_{i}\left(v_{0}\right)$. This gives expressions for $e_{i}, i \notin J$, in terms of the $e_{j}, j \in J$, with constant coefficients, hence similar expressions for $d e_{i}, i \notin J$, in terms of the $d e_{j}, j \in J$, say $d e_{i}=\sum a_{i j} d e_{j}, i \notin J$. Substituting this in the relation (26) above, we find $\omega_{j}=-\sum_{i \notin J} a_{i j} \omega_{i}$ and so

$$
\sum_{i \in I} \omega_{i} \wedge d e_{i}=\sum_{j \in J}\left[\sum_{i \notin J}-a_{i j} \omega_{i}\right] \otimes_{\mathbb{Q}} d e_{j}+\sum_{i \notin J} \omega_{i} \otimes_{\mathbb{Q}}\left[\sum_{j \in J} a_{i j} d e_{j}\right]=0,
$$

which proves the result.

The general case. We now consider the general case where $m \delta_{b}^{\prime} \cdot e_{b} \cdot f_{b} \in$ $\operatorname{im}\left(\mathrm{AJ}_{X}\right) \otimes_{\mathbb{Z}}(\mathbb{R} / \mathbb{Z})^{\otimes 2}$, in contrast to $(23)$. With the above notation, this means that the term $(24)$ vanishes in the quotient of $\left(H^{3}(X, \mathbb{Z}) / H^{3}(X, \mathbb{Z})_{\text {alg }}\right)$ 
$\otimes_{\mathbb{Z}}(\mathbb{R} / \mathbb{Z})^{\otimes 3}$ by $\operatorname{Griff}(X) \otimes_{\mathbb{Z}}(\mathbb{R} / \mathbb{Z})^{\otimes 2}$, where the group

$$
\operatorname{Griff}(X) \subset \frac{H^{3}(X, \mathbb{Z})}{H^{3}(X, \mathbb{Z})_{\mathrm{alg}}} \otimes_{\mathbb{Z}} \mathbb{R} / \mathbb{Z} \cong \frac{J^{3}(X)}{J^{3}(X)_{\mathrm{alg}}}
$$

is countable. This implies the existence of finitely many $c_{n, k} \in \mathbb{R} / \mathbb{Z}$ such that we obtain the following equality in $\left(H^{3}(X, \mathbb{Z}) / H^{3}(X, \mathbb{Z})_{\text {alg }}\right) \otimes_{\mathbb{Z}}(\mathbb{R} / \mathbb{Z})^{\otimes 3}$ :

$$
\begin{aligned}
& m \sum_{n=b_{3}(X)_{\mathrm{alg}}+1}^{b_{3}(X)} \sum_{j=1}^{b_{2}(S)_{\mathrm{new}}} \eta_{n j}(b) \\
& \otimes_{\mathbb{Z}}\left\{\sum_{i=1}^{g} \varrho_{i j}(b) \otimes_{\mathbb{Z}} \psi_{i}(b)-\sum_{i=1}^{g} \chi_{i j}(b) \otimes_{\mathbb{Z}} \phi_{i}(b)\right\} \otimes_{\mathbb{Z}} \delta_{n} \\
&=\sum_{n=b_{3}(X)_{\mathrm{alg}}+1}^{b_{3}(X)} \sum_{k} c_{n, k} \otimes_{\mathbb{Z}} \tau_{n, k} \otimes_{\mathbb{Z}} \delta_{n},
\end{aligned}
$$

where $\tau_{n, k} \in \mathcal{C}^{\infty}(V, \mathbb{R} / \mathbb{Z})^{\otimes 2}$. It is clear that the right hand side vanishes under the triple derivative

$$
d^{3}: \mathcal{C}^{\infty}(V, \mathbb{R} / \mathbb{Z})^{\otimes 3} \rightarrow \wedge^{3} \Omega_{V}^{\mathbb{R}}, \quad f \otimes g \otimes h \mapsto d f \wedge d g \wedge d h .
$$

But if we go back to the construction of $d \delta^{\prime} \wedge d e^{\prime} \wedge d f([\omega])$, we see that it is obtained by a suitable contraction using various Poincaré dualities of $d^{3}\left(\delta^{\prime} \otimes e^{\prime} \otimes f \otimes[\omega]\right)$. It follows that the equality (27) has the same implication on $d \delta^{\prime} \wedge d e^{\prime} \wedge d f([\omega])$ as the equality

$$
\begin{aligned}
& m \sum_{n=b_{3}(X)_{\mathrm{alg}}+1}^{b_{3}(X)} \sum_{j=1}^{b_{2}(S)_{\text {new }}} \eta_{n j}(b) \\
& \otimes_{\mathbb{Z}}\left\{\sum_{i=1}^{g} \varrho_{i j}(b) \otimes_{\mathbb{Z}} \psi_{i}(b)-\sum_{i=1}^{g} \chi_{i j}(b) \otimes_{\mathbb{Z}} \phi_{i}(b)\right\} \otimes_{\mathbb{Z}} \delta_{n}=0
\end{aligned}
$$

considered before (see (24)). So in this case we also conclude that $d \delta^{\prime} \wedge d e^{\prime} \wedge$ $d f([\omega])$ vanishes on $V$.

Putting together Theorem 4.5 and Proposition 5.2 we obtain the following result:

Proposition 5.4. Let $V \subset B$ be a smooth, real-analytic subset such that for all $b \in V$, the product $\delta_{b}^{\prime} \cdot e_{b} \cdot f_{b}$ is torsion in $J^{3}(X)_{\mathrm{AJ}} \otimes_{\mathbb{Z}}(\mathbb{R} / \mathbb{Z})^{\otimes 2}$. Then Mumford's pull-back $M^{*}(\omega)$ of the holomorphic 3-form $\omega$ on $X$ vanishes on $V$.

The other half of the proof of Theorem 5.1 is: 
Proposition 5.5. Assume that $h^{1,0}(X)=b_{4, \operatorname{tr}}(X)=0$, and that the map $\psi_{3}^{3}$ vanishes modulo torsion in $J_{3}^{3}(X)$. Then there exist data

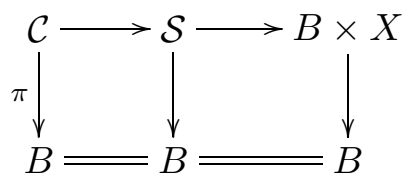

together with sections $s_{i}$ of $\pi$, and integers $m_{i}$, defining a family of 0-cycles $Z_{b}=\sum_{i} m_{i} s_{i}(b)$ homologous to zero on $C_{b}$, with the properties:

(a) There exists a map $\Psi=\left(\Psi_{1}, \Psi_{2}\right): B \rightarrow X \times X$ such that

$$
\left(\operatorname{pr}_{2} \circ j \circ i\right)_{*} Z_{b}=\Psi_{1}(b)-\Psi_{2}(b)
$$

as a 0 -cycle of $X$, for any $b \in B$.

(b) There is a smooth locally closed real-analytic subset $V \subset B$ such that, for all $b \in V$,

$$
\delta_{b}^{\prime} \cdot e_{b} \cdot f_{b}=0 \in\left(J^{3}(X)_{\mathrm{AJ}} \otimes_{\mathbb{Z}}(\mathbb{R} / \mathbb{Z})^{\otimes 2}\right) / T,
$$

where $T$ is the torsion subgroup of $J^{3}(X)_{\mathrm{AJ}} \otimes_{\mathbb{Z}} \mathbb{R} / \mathbb{Z} \otimes_{\mathbb{Z}} \mathbb{R} / \mathbb{Z}$, and

(c) $\Psi_{\mid V}$ is a submersion.

Proof. Since $H^{1,0}(X)=0=H^{2,0}(X)$, the maps $\operatorname{alb}_{X}$ and $\psi_{2}^{3}$ are zero. So $\psi_{3}^{3}$ is defined on the cycles $x-y, x, y \in X$. The assumption that $\psi_{3}^{3}=0$ modulo torsion implies that for any $x, y \in X$ there exist a curve $C$ and a surface $S$ together with morphisms $i: C \rightarrow S$ and $j: S \rightarrow X$, and a cycle $Z$ on $C$ homologous to 0 , such that

1. The cycle $j_{*}\left(i_{*} Z\right)$ is equal to $x-y$ as a 0 -cycle on $X$.

2. The cycle $i_{*} Z$ is Albanese equivalent to 0 on $S$.

3. For some non-zero integer $m$ we have $m \delta_{X, S}^{\prime} \cdot e_{S, C} \cdot f_{C, Z}=0$ in $J^{3}(X)_{\mathrm{AJ}} \otimes_{\mathbb{Z}}(\mathbb{R} / \mathbb{Z})^{\otimes 2}$.

There are countably many algebraic varieties $B_{m}$ parametrizing the data above, except for condition 3 . For each such variety $B_{m}$ we have a morphism

$$
\left(\Psi_{1}^{m}, \Psi_{2}^{m}\right): B_{m} \rightarrow X \times X
$$

given by property 1 .

And for each of these varieties the equations provided by condition 3 are satisfied on a countable union of locally closed real-analytic subsets $V_{n, m}$. Our assumption is that $X \times X$ is filled in by the countable union of the images $\left(\Psi_{1}^{m}, \Psi_{2}^{m}\right)\left(V_{m, n}\right)$. It then follows from Sard's and Baire's theorems that some $\left(\Psi_{1}^{m}, \Psi_{2}^{m}\right)$ must be submersive at some point of some $V_{m, n}$.

This proposition, together with Proposition 5.4, now implies the theorem as follows:

If $\psi_{3}^{3}=0$ modulo torsion, we are in a position to apply Proposition 5.5. On the one hand, by property (a) we see that for any holomorphic 3-form 
$\omega$ on $X$, Mumford's pull-back $M^{*}(\omega)$ is $\Psi_{1}^{*}(\omega)-\Psi_{2}^{*}(\omega)$. On the other hand, by property (b) and by Proposition 5.4, we know that $M^{*} \omega$ vanishes on $V$.

Finally, by property (c) the map $\Psi_{\mid V}: V \rightarrow X \times X$ is submersive, so the vanishing of

$$
\Psi_{1}^{*}(\omega)-\Psi_{2}^{*}(\omega)_{\mid V}=\Psi_{\mid V}^{*}\left(\operatorname{pr}_{1}^{X}(\omega)-\operatorname{pr}_{2}^{X}(\omega)\right)
$$

implies that $\operatorname{pr}_{1}^{X}(\omega)-\operatorname{pr}_{2}^{X}(\omega)$ is zero on an open set of $X \times X$, hence $\omega$ is zero, since it is holomorphic.

In sum, we have shown that, under the assumptions $h^{1,0}(X)=b_{4, \operatorname{tr}}(X)$ $=0$, if $\psi_{3}^{3}=0$ modulo torsion, then there are no holomorphic 3 -forms on $X$, which is just Theorem 5.1.

REMARK 5.6. One can prove that the assumption $h^{1,0}=0$ is not necessary here. Indeed, the set $\mathcal{Z}_{k \text {,alb }} \subset X^{(k)} \times X^{(k)}$ consisting of couples $\left(Z, Z^{\prime}\right)$ such that $\operatorname{alb}_{X}\left(Z-Z^{\prime}\right)=0$ is a Zariski closed algebraic subset of codimension $\leq g:=\operatorname{dim} \operatorname{Alb}(X)$ in $X^{(k)} \times X^{(k)}$. Hence we can apply the argument alluded to in the previous remark to $\mathcal{Z}_{k \text {,alb }}$ for $k \rightarrow \infty$ to conclude that $\operatorname{im~} \psi_{3}^{3}$ is infinite-dimensional if $h^{3,0}(X)$ is non-zero, even if $g \neq 0$.

6. On the non-injectivity of the map $\psi_{3}^{3}$. To conclude, we show that under an additional condition the map $\psi_{3}^{3}$ is not always injective. Consider the special case where the threefold $X$ is the product of a smooth surface $S$ and a smooth curve $C$. We establish a connection between the AbelJacobi map $\operatorname{alb}_{C}=\mu_{C}$ for $C$, the map $\psi_{2}^{2}$ for $S$ and the map $\psi_{3}^{3}$ for $X$. Concretely, we will define the map $k$ indicated below and prove that the following diagram commutes:

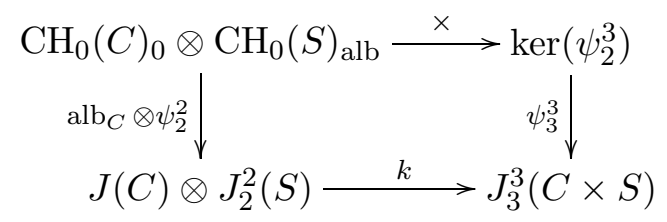

Remark 6.6 explains how this fact can be used to give a counterexample to the demanded injectivity of the map $\psi_{3}^{3}$ based on the one by Voisin for Green's map.

There is a product " $\times$ " for 0 -cycles which factors through rational equivalence (see [Ful, 1.10]):

$$
\mathrm{CH}_{0}(C) \otimes \mathrm{CH}_{0}(S) \rightarrow \mathrm{CH}_{0}(X), \quad \sum_{i} n_{i}\left[c_{i}\right] \times \sum_{j} m_{j}\left[d_{j}\right] \mapsto \sum_{i, j} n_{i} m_{j}\left[c_{i} \times d_{j}\right] .
$$

We begin by showing

Lemma 6.1. The map induced by this product

$$
\mathrm{CH}_{0}(C)_{0} \otimes \mathrm{CH}_{0}(S)_{\mathrm{alb}} \rightarrow \mathrm{CH}_{0}(X)_{\mathrm{alb}}
$$

has its image in $\operatorname{ker}\left(\psi_{2}^{3}\right)$. 
Proof. We will start with the case when $Z=Z_{C} \times Z_{S}$, with $Z_{C}=$ $p-q, p, q \in C$, and $Z_{S} \in \mathrm{CH}_{0}(S)_{\text {alb }}$ arbitrary. Then we can choose the surface $(p \times S) \cup(q \times S) \subset X$ to compute $\psi_{2}^{3}(Z)$, since it contains the support of $Z=(p-q) \times Z_{S}$. Now by construction the map $\psi_{2}^{3}$ is given by pushing forward $\psi_{2}^{2}\left(Z_{S}\right)$ on $p \times S$ and $-\psi_{2}^{2}\left(Z_{S}\right)$ on $q \times S$ via the inclusion map, and this is equivalent to pushing forward $\psi_{2}^{2}\left(Z_{S}\right)$ via the Gysin morphism

$$
j_{p *}-j_{q *}: H^{2}(S) \rightarrow H^{4}(X) \text {. }
$$

But the two push-forward maps in cohomology, $j_{p *}$ and $j_{q *}$, are identical, since they are induced by the homotopic maps $j_{p}$ and $j_{q}$, and it follows that

$$
(p-q) \times Z_{S} \in \operatorname{ker}\left(\psi_{2}^{3}\right) .
$$

The case of an arbitrary cycle $Z \in \mathrm{CH}_{0}(C)_{0} \otimes \mathrm{CH}_{0}(S)_{\text {alb }}$ follows by linearity from this special case.

We now construct the map

$$
k: J(C) \otimes J_{2}^{2}(S) \rightarrow J_{3}^{3}(S \times C),
$$

which will be based on the Künneth inclusion

$$
i: H^{1}(C, \mathbb{Z}) \otimes_{\mathbb{Z}} H^{2}(S, \mathbb{Z}) \hookrightarrow H^{3}(C \times S, \mathbb{Z}) .
$$

By definition,

$$
J_{2}^{2}(S)=\frac{H^{2}(S, \mathbb{Z})_{\mathrm{tr}}^{*} \otimes_{\mathbb{Z}} \mathbb{R} / \mathbb{Z} \otimes_{\mathbb{Z}} \mathbb{R} / \mathbb{Z}}{U_{2}^{2}(S)}
$$

where $H^{2}(S, \mathbb{Z})_{\mathrm{tr}}^{*}=H^{2}(S, \mathbb{Z}) / \mathrm{NS}(S)$, and $J(C)=H^{1}(C, \mathbb{Z}) \otimes_{\mathbb{Z}} \mathbb{R} / \mathbb{Z}$.

Lemma 6.2. The map

$i: H^{1}(C, \mathbb{Z}) \otimes_{\mathbb{Z}} \mathbb{R} / \mathbb{Z} \otimes_{\mathbb{Z}} H^{2}(S, \mathbb{Z}) \otimes_{\mathbb{Z}}(\mathbb{R} / \mathbb{Z})^{\otimes 2} \hookrightarrow H^{3}(C \times S, \mathbb{Z}) \otimes_{\mathbb{Z}}(\mathbb{R} / \mathbb{Z})^{\otimes 3}$ followed by the projections

$$
\pi_{1}: H^{3}(C \times S, \mathbb{Z}) \otimes_{\mathbb{Z}}(\mathbb{R} / \mathbb{Z})^{\otimes 3} \rightarrow J^{3}(C \times S)_{\mathrm{AJ}} \otimes_{\mathbb{Z}}(\mathbb{R} / \mathbb{Z})^{\otimes 2}
$$

and

$$
\pi_{2}: J^{3}(C \times S)_{\mathrm{AJ}} \otimes_{\mathbb{Z}}(\mathbb{R} / \mathbb{Z})^{\otimes 2} \rightarrow \frac{J^{3}(C \times S)_{\mathrm{AJ}} \otimes_{\mathbb{Z}}(\mathbb{R} / \mathbb{Z})^{\otimes 2}}{U_{3}^{3}(C \times S)}=J_{3}^{3}(C \times S)
$$

factors through $J(C) \otimes J_{2}^{2}(S)$.

(We recall that $\pi_{1}$ is the composition of the identifications

$$
\begin{aligned}
H^{3}(C \times S, \mathbb{Z}) \otimes_{\mathbb{Z}}(\mathbb{R} / \mathbb{Z})^{\otimes 3} & =H^{3}(C \times S, \mathbb{Z}) \otimes_{\mathbb{Z}} \mathbb{R} / \mathbb{Z} \otimes_{\mathbb{Z}}(\mathbb{R} / \mathbb{Z})^{\otimes 2} \\
& \cong J^{3}(C \times S) \otimes_{\mathbb{Z}}(\mathbb{R} / \mathbb{Z})^{\otimes 2}
\end{aligned}
$$

and of the projection in $J^{3}(C \times S)_{\mathrm{AJ}}$, that is, modulo the image of the Abel-Jacobi map of $C \times S$.)

We will denote by $k: J(C) \otimes J_{2}^{2}(S) \rightarrow J_{3}^{3}(C \times S)$ the induced map on the higher Jacobians. 
Proof. To begin, we show that for an element $\eta \in \mathrm{NS}(S) \otimes_{\mathbb{Z}}(\mathbb{R} / \mathbb{Z})^{\otimes 2}$ and an element $\gamma \in J(C)=H^{1}(C, \mathbb{Z}) \otimes_{\mathbb{Z}} \mathbb{R} / \mathbb{Z}, i(\gamma \otimes \eta)$ belongs to $\operatorname{im}(\mathrm{AJ}) \otimes_{\mathbb{Z}}$ $(\mathbb{R} / \mathbb{Z})^{\otimes 2}$ and thus vanishes when projected via $\pi_{1}$. In order to see this, note that the class $\eta$, being a Hodge class, induces a morphism of Hodge structures

$$
p_{2}^{*} \eta \cup: H^{1}(C, \mathbb{Z}) \rightarrow H^{3}(C \times S, \mathbb{Z}),
$$

and the corresponding morphism of Jacobians

$$
p_{2}^{*} \eta \cup: J(C) \rightarrow J^{3}(C \times S)
$$

is the Abel-Jacobi map associated to the family of 1-cycles $Z_{C} \times \widetilde{\eta}$ of $C \times S$, where $Z_{C}$ is a 0 -cycle on $C$ of degree zero, and $\widetilde{\eta}$ is a 1 -cycle on $S$ of class $\eta$. Hence the image of $p_{2}^{*} \eta \cup$ (which identifies with the tensor product map via the Künneth decomposition), is contained in the image of the AbelJacobi map of $C \times S$. It follows that for any $\eta=\sum_{i} \eta_{i} \otimes \alpha_{i}, \eta_{i} \in \operatorname{NS}(S)$, $\alpha_{i} \in(\mathbb{R} / \mathbb{Z})^{\otimes 2}$, and any $\gamma \in J(C), i(\gamma \otimes \eta)$ belongs to $\operatorname{im}(\mathrm{AJ}) \otimes_{\mathbb{Z}}(\mathbb{R} / \mathbb{Z})^{\otimes 2}$, which proves our claim.

The second thing to show is that this composite map factors through $J(C) \otimes U_{2}^{2}(S)$, but it can be seen that an element of $J(C) \otimes U_{2}^{2}(S)$ maps to an element of $U_{3}^{3}(X)$ via $\pi_{1} \circ i$ and hence vanishes when projected via $\pi_{2}$. Indeed, these elements are of the form $\operatorname{alb}_{C}(p-q) \otimes \alpha, \alpha$ being obtained by Green's contraction of some $f_{\widetilde{C}, \widetilde{Z}} \cdot e_{\widetilde{C}, S}$, where $\widetilde{Z}$ is a 0 -cycle of $\widetilde{C}$ and $\psi: \widetilde{C} \rightarrow S$ is a morphism such that $\psi_{*} \widetilde{Z}=0$ as a cycle on $S$. We will show below that $\operatorname{alb}_{C}(p-q) \otimes \alpha$ is the Green contraction of $\alpha$ with the Abel-Jacobi invariant of the cycle $\Gamma_{i_{p}}-\Gamma_{i_{q}}$ of $S \times C \times S$. Hence, by definition of $\psi_{3}^{3}$, we find that $\operatorname{alb}_{C}(p-q) \otimes \alpha$ belongs to $U_{3}^{3}(C \times S)$.

Now we can discuss the commutativity of the diagram of the beginning of this section.

Proposition 6.3. The diagram

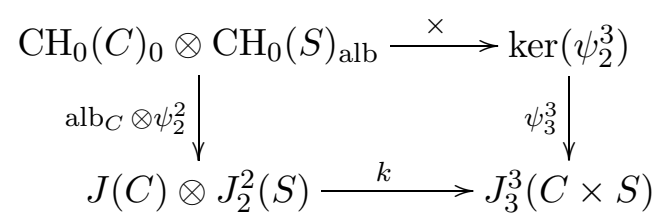

commutes.

Proof. This will result from the following description of the Abel-Jacobi invariant $\delta_{X, p \times S}^{\prime}-\delta_{X, q \times S}^{\prime}$ : Let $\Gamma_{j_{p}}-\Gamma_{j_{q}}$ be the difference of the graphs of the two inclusion maps

$$
j_{p}: S \rightarrow p \times S \subset X \quad \text { and } \quad j_{q}: S \rightarrow q \times S \subset X .
$$


Then by definition $\delta_{X, p \times S}^{\prime}-\delta_{X, q \times S}^{\prime}$ is obtained by projecting

$$
\operatorname{AJ}\left(\Gamma_{j_{p}}-\Gamma_{j_{q}}\right) \in J^{5}(S \times X)
$$

into the appropriate quotient.

By definition of $\psi_{3}^{3}$, since we can see $(p-q) \times Z_{S}$ as the cycle $Z_{S}$ on $p \times S$ and $-Z_{S}$ on $q \times Z_{S}$, we find that $\psi_{3}^{3}\left((p-q) \times Z_{S}\right)$ is obtained as the projection in $J_{3}^{3}(X)$ of Green's contraction of any lifting

$$
\widetilde{\psi_{2}^{2}\left(Z_{S}\right)} \in H^{2}(S, \mathbb{Z}) \otimes_{\mathbb{Z}}(\mathbb{R} / \mathbb{Z})^{\otimes 2}
$$

of $\psi_{2}^{2}\left(Z_{S}\right)$ and of the real version of $\delta_{X, p \times S}^{\prime}-\delta_{X, q \times S}^{\prime}$. Since the latter is obtained as a projection of the (real version of the) Abel-Jacobi invariant of $\Gamma_{j_{p}}-\Gamma_{j_{q}}$, we start by computing $\operatorname{AJ}\left(\Gamma_{j_{p}}-\Gamma_{j_{q}}\right) \in J^{5}(S \times X)$. Let $\Delta_{S} \subset S \times S$ be the diagonal; we see immediately that

$$
\Gamma_{j_{p}}-\Gamma_{j_{q}}=p_{13}^{*}\left(\Delta_{S}\right) \cdot p_{2}^{*}(p-q)
$$

as a cycle of $S \times C \times S$.

Now by (29) we have the following formula for its Abel-Jacobi image in $J^{5}(S \times X)=J\left(H^{5}(S \times X)\right):$

$$
\operatorname{AJ}\left(\Gamma_{j_{p}}-\Gamma_{j_{q}}\right)=p_{13}^{*}\left(\left[\Delta_{S}\right]\right) \cdot p_{2}^{*}\left(\operatorname{alb}_{C}(p-q)\right) .
$$

We project this invariant via

$$
\text { pr : } J\left(H^{5}(S \times X)\right) \rightarrow J\left(H^{2}(S) \otimes H^{3}(X)\right) ;
$$

from (30), by analyzing Künneth types, it follows that

$$
\operatorname{pr}\left(\operatorname{AJ}\left(\Gamma_{j_{p}}-\Gamma_{j_{q}}\right)\right)=p_{13}^{*}\left(\left[\Delta_{S}\right]_{(2,2)}\right) \cdot p_{2}^{*}\left(\operatorname{alb}_{C}(p-q)\right),
$$

where $\left[\Delta_{S}\right]_{(2,2)}$ is the $(2,2)$-Künneth component of $\Delta_{S}$. Now since $\left[\Delta_{S}\right]_{(2,2)}$ is a Hodge class, the identifications

$$
\begin{gathered}
J(C) \cong H^{1}(C, \mathbb{Z}) \otimes_{\mathbb{Z}} \mathbb{R} / \mathbb{Z}, \\
J^{5}(S \times C \times S) \cong H^{5}(S \times C \times S, \mathbb{Z}) \otimes_{\mathbb{Z}} \mathbb{R} / \mathbb{Z}
\end{gathered}
$$

are compatible with the cup product with $p_{13}^{*}\left[\Delta_{S}\right]_{(2,2)}$ (which via the Künneth decomposition is given by the tensor product with $\left[\Delta_{S}\right]_{(2,2)}$.) Hence we have proved

Lemma 6.4. The invariant $\mathrm{AJ}\left(\Gamma_{j_{p}}-\Gamma_{j_{q}}\right)$, viewed as an element of

$$
H^{2}(S, \mathbb{Z}) \otimes_{\mathbb{Z}} H^{1}(C, \mathbb{Z}) \otimes_{\mathbb{Z}} H^{2}(S, \mathbb{Z}) \otimes_{\mathbb{Z}} \mathbb{R} / \mathbb{Z},
$$

is equal to

$$
p_{13}^{*}\left[\Delta_{S}\right]_{(2,2)} \cup p_{2}^{*} f_{C, p-q} .
$$

(Here the cup product identifies with a tensor product, and the element $f_{C, p-q} \in H^{1}(C, \mathbb{Z}) \otimes_{\mathbb{Z}} \mathbb{R} / \mathbb{Z}$ is the real version of $\operatorname{alb}_{C}(p-q)$.) 
In order to compute $\psi_{3}^{3}(Z)$, we have to make Green's contraction of $\widetilde{\psi_{2}^{2}\left(Z_{S}\right)}$ and $\operatorname{AJ}\left(\Gamma_{j_{p}}-\Gamma_{j_{q}}\right)$. We now use the following lemma whose proof is obvious:

Lemma 6.5. The map $H^{2}(S, \mathbb{Z}) \rightarrow H^{2}(S, \mathbb{Z})$, given by contraction with the class $\left[\Delta_{S}\right]_{(2,2)} \in H^{2}(S, \mathbb{Z}) \otimes_{\mathbb{Z}} H^{2}(S, \mathbb{Z})$ using Poincaré duality, is equal to the identity.

It follows from this lemma that Green's contraction of $\widetilde{\psi_{2}^{2}\left(Z_{S}\right)}$ with

$$
p_{13}^{*}\left[\Delta_{S}\right]_{(2,2)} \cup p_{2}^{*} f_{p-q, C}
$$

is equal to

$$
f_{C, p-q} \otimes \widetilde{\psi_{2}^{2}\left(Z_{S}\right)} \in H^{1}(C, \mathbb{Z}) \otimes_{\mathbb{Z}} H^{2}(S, \mathbb{Z}) \otimes_{\mathbb{Z}}(\mathbb{R} / \mathbb{Z})^{\otimes 3} .
$$

This proves the commutativity of the diagram (28).

To conclude this section, we point out the following fact:

REMARK 6.6. One knows that the map $\psi_{2}^{2}$ is not injective (see [Voi3, Section 2]). The description of the map $\psi_{3}^{3}$ just given in this special case shows that it is not injective if we assume that the following statement is true:

Let $Z_{S}$ be a 0 -cycle on a surface $S$ which is not rationally equivalent to 0 . Then there exist a curve $C$ and a 0 -cycle $Z_{C}$ of degree 0 on $C$ such that $Z_{S} \times Z_{C}$ is not rationally equivalent to 0 on $C \times S$.

\section{References}

[Bei] A. Beilinson, Height pairing between algebraic cycles, in: Current Trends in Arithmetical Algebraic Geometry, K. Ribet (ed.), Contemp. Math. 67, Amer. Math. Soc., Providence, 1987, 1-24.

[Car1] J. Carlson, Extensions of mixed Hodge structures, in: Géométrie algébrique (Angers, 1979), A. Beauville (ed.), Sijthoff \& Noordhoff, 1980, 107-127.

[Car2] -, The geometry of the extension class of a mixed Hodge structure, in: Proc. Sympos. Pure Math. 46, Amer. Math. Soc., 1987, 199-222.

[EZ] F. El Zein and S. Zucker, Extendability of normal functions associated to algebraic cycles, in: Topics in Transcendental Algebraic Geometry, Ph. Griffiths (ed.), Ann. of Math. Stud. 106, Princeton Univ. Press, 1984, 269-288.

[EV] H. Esnault and E. Viehweg, Deligne-Beilinson cohomology, in: Beilinson's Conjectures on Special Values of $L$-functions, M. Rapoport et al. (eds.), Perspect. Math. 4, Academic Press, 1988, 43-91.

[Ful] W. Fulton, Intersection Theory, Ergeb. Math. Grenzgeb. 2, Springer, 1998.

[Gre1] M. Green, What comes after the Abel-Jacobi map?, preprint, 1997, 54 pp.

[Gre2] -, Higher Abel-Jacobi maps, in: Proc. ICM Berlin 1998, Doc. Math. 1998, Extra Vol. II, 267-276.

[GMV] M. Green, J. Murre and C. Voisin, Algebraic Cycles and Hodge Theory, Lecture Notes in Math. 1594, Springer, 1993. 
[Mum] D. Mumford, Rational equivalence of 0-cycles on surfaces, J. Math. Kyoto Univ. 9 (1969), 195-204.

[Mur] J. Murre, On the motive of an algebraic surface, J. Reine Angew. Math. 409 (1990), 190-204.

[Voi1] C. Voisin, Hodge Theory and Complex Algebraic Geometry I, Cambridge Stud. Adv. Math. 76, Cambridge Univ. Press, Cambridge, 2003.

[Voi2] - Hodge Theory and Complex Algebraic Geometry II, Cambridge Stud. Adv. Math. 77, Cambridge Univ. Press, Cambridge, 2003.

[Voi3] —, Some results on Green's higher Abel-Jacobi map, Ann. of Math. 149 (1999), 451-473.

Mathematisches Institut der Universität Erlangen

Bismarckstrasse $1 \frac{1}{2}$

91054 Erlangen, Germany

E-mail: lorenz.schneider@btinternet.com

Received 21 January 2005;

in revised form 15 June 2005 